\title{
A review of toxicity testing protocols and endpoints with Artemia spp.
}

\author{
G. Libralato ${ }^{\mathrm{a}, *}$, E. Prato $^{\mathrm{b}}$, L. Migliore ${ }^{\mathrm{c}}$, A.M. Cicero $^{\mathrm{d}}$, L. Manfra ${ }^{\mathrm{d}, \mathrm{e}}$ \\ a Department of Environmental Sciences, Informatics and Statistics, University Ca' Foscari Venice, Via Torino 155, 30172 Mestre, Venice, Italy \\ ${ }^{\mathrm{b}}$ Institute for the Coastal Marine Environment, National Research Council (CNR IAMC), Via Roma 3, 74100 Taranto, Italy \\ c Department of Biology, University Tor Vergata, Via della Ricerca Scientifica, 00133 Rome, Italy \\ d Italian Institute for Environmental Protection and Research, Via Vitaliano Brancati 60, 00144 Rome, Italy \\ e Stazione Zoologica Anton Dohrn, Villa Comunale, 80121 Naples, Italy
}

\section{A R T I C L E I N F O}

\section{Article history:}

Received 6 March 2016

Received in revised form 3 April 2016

Accepted 5 April 2016

\section{Keywords:}

Artemia

Toxicity test methods

Hatching

Biomarker

Behavioral endpoints

Immobilization/mortality and survival

\begin{abstract}
A B S T R A C T
Artemia spp. is an historically popular biological model still requiring an official internationally based standardization. Several endpoints are currently available. Short-term acute endpoints include biomarker (acetylcholinesterase; heat stress proteins; lipid peroxidation; thiobarbituric acid reactive substances; thioredoxin reductase; glutathione-peroxidase; glutathione $S$-transferase; glutathione reductase; aldehyde dehydrogenase; and adenylpyrophosphatase and Fluotox), hatching (dry biomass, morphological disorders and size), behavioral (swimming speed and path length), teratogenicity (growth), and immobilization (meaning mortality after 5-30s observation). Long-term chronic tests focus on growth, reproduction and survival or mortality after 7-28 d exposure from larval to adulthood stage. We analyzed each test looking at its endpoint, toxicant and experimental design including replicates, exposure time, number of exposed cysts or organisms and their relative life stage, exposure conditions during hatching and testing (salinity, pH, light intensity, aeration dilution media, and food supply), type of testing chambers, and quality assurance and quality control criteria. Similarities and differences between the identified approaches were highlighted. Results evidenced that hatching $24 \mathrm{~h}$ short-term and $14 \mathrm{~d}$ longterm mortality are the most promising Artemia spp. protocols that should go forward with international standardization.
\end{abstract}

(c) 2016 Elsevier Ltd. All rights reserved.

\section{Introduction}

The adoption and implementation of the European legislation about the Registration, Evaluation, Authorization, and Restriction of Chemicals (REACH) (EC, 2006) required several additional ecotoxicity data promoting the decrease of vertebrates used in toxicity testing encouraging alternative strategies with invertebrates, plants as well as organ, tissue, and cell cultures (Dvorak et al., 2012). During the last 50 years, various invertebrates were assessed to investigate their sensitivity to many physical and chemical agents for their possible use as pre-screening or screening models. Internationally, Artemia spp. brine shrimps (Crustacea, Branchiopoda, Anostraca), commonly known also as sea monkeys, are one of the most frequently used species for toxicity testing (Van Steertegem and Persoone, 1993a,b).

Artemia spp. is a major taxon in many hypersaline biotypes throughout the world feeding primarily on phytoplankton and

\footnotetext{
* Corresponding author.

E-mail address: giovanni.libralato@unive.it (G. Libralato).
}

being an important primary consumer (Persoone and Sorgeloos, 1980; Vanhaecke et al., 1987; Triantaphyllidis et al., 1998). They are of economical importance being used in aquaculture and in aquariology. They also act as an efficiency and productivity stimulator for salt production in solar salt works (Jones et al., 1981; Migliore et al., 1997; Treece, 2000).

The main advantages of using brine shrimps in toxicity testing are: (i) rapidity (i.e. $28-72 \mathrm{~h}$ from hatching to the first endpoint); (ii) cost-effectiveness;(iii) the availability of nauplii hatched from commercial durable cysts (eggs) (i.e. homogeneity of the population, availability all year-round without the necessity of culturing) (Nunes et al., 2006a; Manfra et al., 2012). Other advantages are: (i) good knowledge of its biology and ecology; (ii) easy manipulation and maintenance under laboratory conditions; (iii) small body size allowing accommodation in small beakers or microplates; (iv) high adaptability to various testing conditions (Nunes et al., 2006a; Kokkali et al., 2011). Conversely, several criticisms about Artemia spp. sensitivity were presented by a learning-by-doing approach (Libralato et al., 2010a,b; Libralato, 2014). For example, the cysts' production can reflect the occurrence of genetic variation caused by crustaceans' geographical origin that is rarely known (Migliore 
et al., 1997), although certified cysts are usually utilized in toxicity testing. Their origin can have consequences on the growth, survival and reproduction of Artemia spp. specimens considering especially salinity and temperature (Vanhaecke and Sorgeloos, 1989; Triantaphyllidis et al., 1995).

Artemia spp. nauplii were used to test the toxicity of a wide range of chemicals such as arsenic (As) (Brix et al., 2003), cadmium (Cd) (Kissa et al., 1984; Hadjispyrou et al., 2001; Sarabia et al., 1998a, 2002, 2006; Brix et al., 2006; Leis et al., 2014), chromium (Cr) (Hadjispyrou et al., 2001; Leis et al., 2014), cobalt (Kissa et al., 1984), copper (Cu) (Browne, 1980; Jorgensen and Jensen, 1977; Brix et al., 2006), mercury (Hg) (Sarabia et al., 1998b; Leis et al., 2014), nickel (Kissa et al., 1984), tin (Sn) (Hadjispyrou et al., 2001), zinc (Zn) (Brix et al., 2006; Garaventa et al., 2010), potassium permanganate, potassium dichromate, and silver nitrate (Boone and Baas-Becking, 1931; Vanhaecke et al., 1980), antibiotic drugs (Migliore et al., 1993a,b, 1997), engineered nanomaterials (Libralato, 2014; Minetto et al., 2014; Corsi et al., 2014; Callegaro et al., 2015), nano-sized polystyrene (Bergami et al., 2016), asbestos (Stewart and Schurr, 1980), phenolic compounds (Guerra, 2001), ethanolamines (Libralato et al., 2010a) and trace elements (Petrucci et al., 1995), triazine herbicides, insecticides, pesticides (Kuwabara et al., 1980; Varó et al., 1997, 2002), acrylonitrile (Tong et al., 1996), carbammates (Barahona and Sánchez-Fortún, 1999), phthalates, antifouling agents (Grosch, 1980; Persoone and Castritsi-Catharios, 1989a,b; Okamura et al., 2000; Castritsi-Catharios et al., 2007, 2013, 2014; Koutsaftis and Aoyama, 2007), pharmaceuticals (Xu et al., 2015), anticorrosive agents (Tornambè et al., 2012; Manfra et al., 2015a, 2016), oil (Trieff, 1980) and oil dispersants (Zillioux et al., 1973; Savorelli et al., 2007), various plant extracts (Cáceres et al., 1998), toxins (Granade et al., 1976; Medlyn, 1980; Vezie et al., 1996; Beattie et al., 2003) and environmental matrices such as wood leachates (Libralato et al., 2007), wastewater (Krishnakurmar et al., 2007; Libralato et al., 2010b), seawaters (Manfra et al., 2011) and marine discharges (Manfra et al., 2010).

Currently, various toxicity tests with Artemia spp. are available including short-term and long-term methods. Short-term toxicity tests are more frequently used, some long-term protocols have been developed in the last 10 years, but none of them is an internationally standardised method like International Standard Organization (ISO), American Society for Testing and Materials (ASTM) or Organization for Economic Co-operation and Development (OECD). Methods for testing immobilization/mortality were standardised only in Italy by the Italian Agency for Environmental Protection and Italian Institute for Water Research (APAT IRSACNR) and Italian Agency for Standardization in the Chemical sector (Unichim). Despite the frequent and widespread use of Artemia spp. in toxicity testing, the harmonization of protocols followed by international standardization activities is still lacking, and intercalibration exercises are urgently necessary (Libralato, 2014).

The aim of this review paper is to collect, organize, select and discuss the existing knowledge about Artemia spp. methods for toxicity testing including both short- and long-term bioassays and organism hatching and maintenance conditions providing tips for protocols definition, implementation and standardization.

\section{Hatching of cysts}

Artemia spp. cyst hatching conditions can vary greatly as reported in Table S1 $(n=42)$. This can result in a different evaluation of cysts/nauplii sensitivity, although the first factor that can affect organism sensitivity is the geographical origin of cysts. Other species are commercially available, but their sensitivity must be evaluated on Vanhaecke et al. (1980) a case-by-case basis if no certification or traceability is available (Guzzella, 1997).
The second key point is to start the toxicity tests with nauplii belonging to the same class of age because some stages are more sensitive than others (i.e. Instar I stage is less sensitive than Instar II-III stage) (Sorgeloos et al., 1978). Cyst hatching occurred $24 \mathrm{~h}$ before starting the toxicity test (Instar I stage) in 10 papers (Vanhaecke et al., 1981; Barahona et al., 1994; Brix et al., 2003, 2004; Caldwell et al., 2003; Venkateswara Rao et al., 2007; Garaventa et al., 2010; Bustos-Obregon and Vargas, 2010) and $30 \mathrm{~h}$ in 3 papers (Persoone et al., 1993; Guerra, 2001; Koutsaftis and Aoyama, 2007). Most authors used $48 \mathrm{~h}$ old larvae hatched at $25 \pm 2{ }^{\circ} \mathrm{C}$ involving during the exposure larvae at Instar II-III (Guzzella, 1997; Hadjispyrou et al., 2001; APAT and IRSA-CNR, 2003; Favilla et al., 2006; Libralato et al., 2007; Savorelli et al., 2007; Pimentel et al., 2009; Manfra et al., 2011, 2010; Kokkali et al., 2011; Prato et al., 2011; Manfra et al., 2012; Tornambè et al., 2012; Leis et al., 2014; Veni and Pushpanathan, 2014; Manfra et al., 2015a,b, 2016; Rotini et al., 2015).

Either artificial or natural seawaters are used as hatching media. Artificial media are more frequently used because salt blends to create the ideal saltwater are commercially available (i.e. Crystal Sea ${ }^{\circledR}$ Marinemix, Forty Fathoms ${ }^{\circledR}$, Coral Reef Red Sea Salt ${ }^{\circledR}$, Instant Ocean $^{\circledR}$ ) or are provided along with toxkits (Artoxkit, 2014). The required seawater can be obtained dissolving these salts in distilled and deionized water.

Cysts are usually incubated at between $18-28^{\circ} \mathrm{C}$ and largely $35 \%$ salinity. The values of $\mathrm{pH}$ vary between $7.5-9.0$ and $\mathrm{pH}$ should not be lower than 7 to obtain good hatching (Vanhaecke et al., 1980). During the hatching process, seawater is sometimes aerated by an air pump being the medium adequate aeration a prerequisite to obtain a successful hatching (Vanhaecke et al., 1980). Manfra et al. (2016) proposed an oxygen saturation level $>60 \%$. The hatching phase occurred in presence of light (1000-4000 lux) (Varó et al., 2002; Artoxkit, 2014) or partly in darkness like just $1 \mathrm{~h}$ of light during $48 \mathrm{~h}$ exposure (Guzzella, 1997; Unichim, 2012) or 12-16 h of light during $24 \mathrm{~h}$ exposure (Garaventa et al., 2010; Gambardella et al., 2014).

The hatching efficiency needs to be considered, but only Guzzella (1997) proposed a threshold evaluating cysts hatching efficiency that should be $>90 \%$ in $\leq 32 \mathrm{~h}$.

Two main cyst-hatching procedures were identified on the basis of Artoxkit (2014) or Unichim (2012). Artoxkit (2014) suggests hatching cysts $30 \mathrm{~h}$ before toxicity testing. Cysts are transferred into a Petri dish with $9 \mathrm{~mL}$ of seawater prepared with the toxkit salts and gently swirled to distribute them evenly. The Petri dish is exposed to a light source (1000-4000 lux) for $30 \mathrm{~h}$. According to Unichim (2012), cyst hatching starts $48 \mathrm{~h}$ before the toxicity test. Seawater is used as hatching medium. Artemia cysts $(100 \mathrm{mg})$ are transferred into a Petri dish with $12 \mathrm{~mL}$ of seawater exposed at 1000-4000 lux for $1 \mathrm{~h}$ and for $23 \mathrm{~h}$ in darkness at $25 \pm 1{ }^{\circ} \mathrm{C}$. After $24 \mathrm{~h}$, hatched nauplii are pipetted to a new Petri dish for moulting containing new fresh seawater that is incubated for $24 \mathrm{~h}$ at $25 \pm 1{ }^{\circ} \mathrm{C}$ in the dark allowing larvae to reach II or III Instar stage.

Sometimes, Artemia embryos were sterilized and dechorionated before use, as in the protocols for aquaculture purposes (Sorgeloos et al., 1977). Dechorionated cysts are Artemia embryos enveloped only by the embryonic cuticle and the outer cuticular membrane (Léger et al., 1986). The technique was set up by Nakanishi et al. (1962), which used a chilled diluted antiformin solution to dissolve the chorion. Morris and Afzelius (1967) improved this technique, which allows removing the outer part of the shell without affecting embryo viability. The temperature of the medium must be kept below $40^{\circ} \mathrm{C}$ to maintain the maximal hatching efficiency (Sorgeloos et al., 1977). The use of antibiotics (i.e. penicillin (50 units $/ \mathrm{mL}$ ), streptomycin $(50 \mu \mathrm{g} / \mathrm{mL})$ and sodium tetraborate $(0.2 \% \mathrm{w} / \mathrm{v}))$ was highlighted by Bagshaw et al. (1986) and Brix et al. (2006). Embryos 


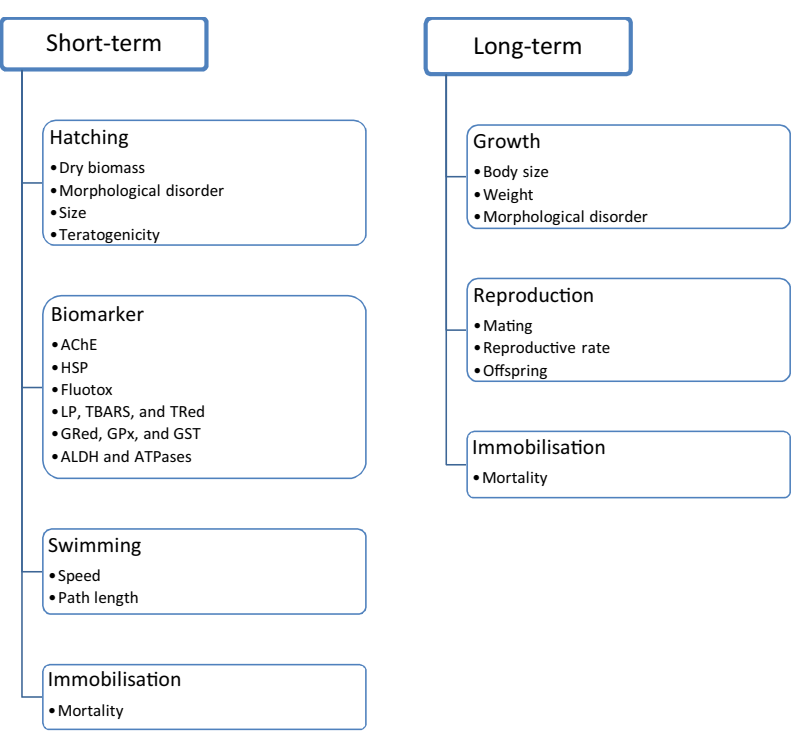

Fig. 1. Summary of Artemia short- and long-term toxicity tests $(\mathrm{AChE}=$ AcetylCholinEsterase; $\mathrm{HSP}=$ heat stress proteins; $\mathrm{LP}=$ lipid peroxidation; TBARS = thiobarbituric acid reactive substances; TRed =thioredoxin reductase; $\mathrm{GPx}=$ glutathione-peroxidase; $\mathrm{GST}=$ glutathione $S$-transferase; GRed = glutathione reductase; $\mathrm{ALDH}=$ aldehyde dehydrogenase; and ATPases = AdenylTriPhosphatase).

were hydrated in sterile seawater containing antibiotics prior to toxicity test in order to prevent potential bacterial infections.

\section{Toxicity tests}

We decided to cluster toxicity tests with Artemia spp. as shortterm acute and long-term chronic bioassays (Fig. 1) considering the life span of Artemia spp. varying between 2-4 months depending on salinity, temperature and species-specific characteristics (Browne et al., 1991). Acute toxicity tests assess the effects based on relatively high exposure concentrations (i.e. $\mathrm{mg} / \mathrm{L}$ ) for no more than $96 \mathrm{~h}$. Toxicity is generally expressed as lethal concentration causing the death of $50 \%$ of the group of test animals $\left(\mathrm{LC}_{50}\right)$, but also hatching and swimming behavior impairment. The analysis was carried out considering sub-lethal (acute cyst hatching test, biomarkers, teratogenicity test, acute larvae behavioral test) and lethal endpoints.

Chronic toxicity tests investigate the long-term exposure from weeks (e.g. 2-4 weeks) up to the whole life cycle of Artemia spp. at relatively low concentrations $(\mu \mathrm{g} / \mathrm{L})$ (Pane et al., 2012). Toxicity is expressed as the no observed effect concentration (NOEC) and lowest observed effect concentration (LOEC) considering survival, growth and reproduction as endpoints (Brix et al., 2003, 2004; Savorelli et al., 2007; Manfra et al., 2012; Unichim, 2012).

\subsection{Short-term toxicity test}

Acute endpoints generally investigate the extent of lethality (Persoone et al., 1993; Guzzella, 1997; Artoxkit, 2014) (Table S2) or other significant impacts on Artemia spp. such as hatching (Table 1), growth (Migliore et al., 1997; Sarabia et al., 2008), and swimming (Garaventa et al., 2010; Gambardella et al., 2014) (Table 2). In most of the cases, readings are carried out just at the end of the exposure period, keeping to a minimum continuous and/or periodic observations of single animals in a non-endpoint perspective. Generally, long-term toxicity tests require the periodical recording of death animals depicting how contact time may contribute to the final evolution of effects.

Artemia 'death' (i.e. mortality or lethality) presented various definitions generating potential misunderstanding about the observation of moribund or really dead larvae. Generally, mortality evaluation is carried out with a stereomicroscope by eye, but computer based analysis systems are available to decide whether the monitored Artemia nauplius is alive or dead (Garaventa et al., 2010; Alyuruk et al., 2013). Widdows (1998) stated that Artemia, and other crustaceans, can lie on the bottom of the test container, but still occasionally move an appendage. 'Immobility' may therefore be an easier state to define. Anyhow, differences in how the immobility state is defined exist. Garaventa et al. (2010) considered nauplii as dead when they are completely motionless or do not change their barycenter position, or no appendage movement occurs for $5 \mathrm{~s}$. Zulkifli et al. (2014) reported that nauplii are dead if they do not show any movement after $10 \mathrm{~s}$ observation, while Alyuruk et al. (2013) prolonged the observation period up to $30 \mathrm{~s}$.

\subsubsection{Acute cyst hatching test}

Since 1980s' cysts and their embryonic development were used in ecotoxicology as summarized in Table 1 . The hatching toxicity test was developed to assess the effect of toxicants having different composition and mode of action like metals (Bagshaw et al., 1986; Brix et al., 2006; MacRae and Pandey, 1991; Rafiee et al., 1986; Jorgensen and Jensen, 1977; Sarabia et al., 1998a,b, 2003, 2008), organic compounds (Kuwabara et al., 1980; Alyürüc and Çavas, 2013; Rotini et al., 2015; Vismara, 1998), antibiotic drugs (Migliore et al., 1993a,b, 1997) or cell extracts from invertebrates, macro- and microalgae (Caldwell et al., 2003; Carballo et al., 2002) as well as stress events generated by salinity and temperature (Vanhaecke and Sorgeloos, 1989). Hatching toxicity tests are static or semi-static lasting between $24 \mathrm{~h}$ and $96 \mathrm{~h}$. They investigate the reduced emergence of nauplii from cysts/eggs when exposed to toxicants compared to negative controls. Results of 'cyst based' toxicity tests with metals, using different procedures, produced apparently inconsistent data. Bagshaw et al. (1986) tested fully hydrated Artemia cysts (20-30 organisms per replicate) at $28^{\circ} \mathrm{C}$ and 36 Practical Salinity Unit (PSU) under constant illumination and shaking, evidencing that encysted embryos are more sensitive to $\mathrm{Cd}$ and $\mathrm{Zn}$ than pre-nauplius larvae. Cadmium $(0.1 \mu \mathrm{M})$ retarded the hatching and development of larvae, while higher concentrations blocked the hatching phase almost completely. Zinc was less toxic than Cd, but it caused similar effects. Bagshaw et al. (1986) protocol was used by Rafiee et al. (1986) and Brix et al. (2006) confirming both the toxicity of $\mathrm{Cd}$ and $\mathrm{Cu}$ (Artemia franciscana), respectively. MacRae and Pandey (1991) showed that Artemia franciscana $\mathrm{Cu}$ and $\mathrm{Pb}(0.1 \mu \mathrm{M})$ toxicity was similar to $\mathrm{Cd}$ reducing the rate and extent of larval development; $\mathrm{Zn}$ was less toxic than $\mathrm{Cd}, \mathrm{Pb}$ and Ni. Sarabia et al. (1998a,b, 2003, 2008), using hydrated cysts of Artemia parthenogenetica (10 organisms per replicate) did not highlight any adverse effects of $\mathrm{Cd}, \mathrm{Hg}$ and $\mathrm{Zn}$ to nauplii emergence. The difference in Artemia spp. sensitivity to metals was probably due to the differences in testing temperature and interspecific species sensitivities. In 1931, Boon and Baas-Becking observed that temperature affected emergence and hatching. Vanhaecke and Sorgeloos (1989) demonstrated that the incubation temperature between 25 and $37^{\circ} \mathrm{C}$ can significantly affect the hatching percentage of Artemia cysts even within geographical strains of both $A$. franciscana and $A$. partenogenetica. In Artemia spp., temperature is known to profoundly influence chemicals' effects. In particular, low temperature reduced toxicity of organo-metallic compounds in $A$. franciscana: $\mathrm{Cu}$ pyrithione and Diuron toxicity at $<25^{\circ} \mathrm{C}$ were significantly reduced or eliminated, respectively (Koutsaftis and Aoyama, 2008). Thus temperature is a variable of extreme interest to be considered while standardizing from hatching, larvae maintenance up to the end of the exposure to treatments. The use of a full range of temperatures, especially during toxicity testing, may drastically increase the ecological relevance of data. 
Artemia spp. behavioural toxicity tests.

\begin{tabular}{|c|c|c|c|c|c|c|c|c|c|c|c|c|c|c|c|c|c|c|c|c|}
\hline References & Test & \begin{tabular}{l|l} 
Toxicant \\
\end{tabular} & $\begin{array}{l}\text { Concentration } \\
(\mathrm{mg} / \mathrm{L})\end{array}$ & $\begin{array}{l}\text { Exposure } \\
\text { (h) }\end{array}$ & $\begin{array}{l}\begin{array}{l}\text { Cysts } \\
\text { (mg) }\end{array} \\
\text { (mg) }\end{array}$ & Endpoint & $\begin{array}{l}\mathrm{T} \\
\left({ }^{\circ} \mathrm{C}\right)\end{array}$ & $\begin{array}{l}\text { Salinity } \\
(\%)\end{array}$ & & $\begin{array}{l}\text { Test } \\
\text { light } \\
(\mathrm{l} x)\end{array}$ & Aereation & $\begin{array}{l}\text { Dilution } \\
\text { medium }\end{array}$ & $\begin{array}{l}\text { Food } \\
\text { (cell/mL) }\end{array}$ & $\begin{array}{l}\text { Organisms } \\
\text { per } \\
\text { chamber }\end{array}$ & Replicates & $\begin{array}{l}\text { Test vessel } \\
(\mathrm{mL})\end{array}$ & $\begin{array}{l}\text { Test } \mathrm{mL} \\
\end{array}$ & QC & $\begin{array}{l}\text { Reference } \\
\text { Toxi- } \\
\text { cant } \\
\end{array}$ & NOEC/LOEC/LC $50 / \mathrm{EC}_{50}$ \\
\hline $\begin{array}{l}\text { Alyuruk et al. } \\
\text { (2013) }\end{array}$ & $\mathrm{s}$ & $\begin{array}{l}\mathrm{K}_{2} \mathrm{Cr}_{2} \mathrm{O}_{7}, \\
\text { p-coumaric } \\
\text { acid }\end{array}$ & 7-30-70-100 & 24 & $\begin{array}{ll}\text { n.a. } \\
\text { n. }\end{array}$ & $\begin{array}{l}\text { SW velocity and } \\
\text { paths covered by } \\
\text { nauplii }\end{array}$ & n.a. & 35 & n.a. & $\begin{array}{l}\text { Low } \\
\text { light }\end{array}$ & n.a. & $\begin{array}{l}\begin{array}{l}\text { Saline } \\
\text { water }\end{array} \\
\end{array}$ & n.a. & $5-8 / 20 \mu \mathrm{L}$ & n.a. & $\mathrm{MP}(96) 130 \mu \mathrm{L}$ & 0.2 & n.a. & $\mathrm{K}_{2} \mathrm{Cr}_{2} \mathrm{O}_{7}$ & $\begin{array}{l}\text { \% survival and velocities for } \\
\text { each concentration and paths }\end{array}$ \\
\hline $\begin{array}{r}\text { Davenport and } \\
\text { Healy (2006) }\end{array}$ & $\mathrm{s}$ & salinity & $8.5-250$ & 24 & n.a. & SW speed & 23 & $\begin{array}{l}8.5- \\
250\end{array}$ & n.a. & n.a. & $\begin{array}{l}\text { air- } \\
\text { saturated }\end{array}$ & NSW & No food & n.a. & n.a. & $\begin{array}{l}\text { Flat-faced } \\
\text { transparent } \\
\text { polystyrene } \\
\text { canted-neck } \\
\text { culture flasks }\end{array}$ & 50 & n.a. & n.a. & n.a. \\
\hline $\begin{array}{l}\text { Gambardella } \\
\text { et al. (2014) }\end{array}$ & $\mathrm{s}$ & $\begin{array}{l}\mathrm{SnO}_{2}, \mathrm{CeO}_{2} \text { and } \\
\mathrm{Fe}_{3} \mathrm{O}_{4} \mathrm{NPs}^{-}\end{array}$ & 10-100-1000 & 48 & 500 & SW and $M$ & 20 & 37 & n.a. & $\begin{array}{l}16 \mathrm{~h} \\
\text { light }\end{array}$ & n.a. & ASW & n.a. & $10-15$ & 9 & $\begin{array}{l}25 \\
\text { compartment } \\
\text { square Petri } \\
\text { dish }\end{array}$ & 1 & n.a. & n.a. & 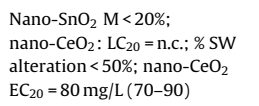 \\
\hline $\begin{array}{l}\text { Garaventa et al. } \\
(2010)\end{array}$ & $\mathrm{s}$ & $\begin{array}{l}\text { 1. zinc } \\
\text { pyrithione; } 2 \text {. } \\
\text { Macrotrol }{ }^{\circledR} \\
\text { MT-200; } 3 \text {. } \\
\text { Eserine }\end{array}$ & $\begin{array}{l}1.0,1-1-10-100 ; 2 . \\
1.5-3-6-9-12 ; 3 \\
0.1-1-10-100\end{array}$ & $24-48$ & 500 & SW and $M$ & 20 & 37 & n.a. & $\begin{array}{l}16 \mathrm{~h} \\
\text { light }\end{array}$ & n.a. & ASW & n.a. & $10-15$ & 9 & Petri dish & 1 & n.a. & n.a. & 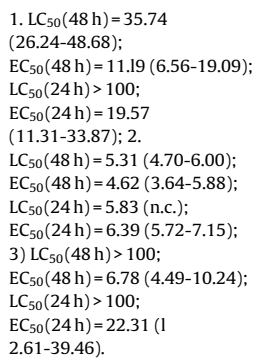 \\
\hline $\begin{array}{l}\text { Huang et al. } \\
\text { (2015) }\end{array}$ & s & $\begin{array}{l}\text { 1. } \mathrm{K}_{2} \mathrm{Cr}_{2} \mathrm{O}_{7} ; 2 . \\
\mathrm{Cd}\left(\mathrm{NO}_{3}\right)_{2}\end{array}$ & 1. $0-100 ; 2.0-1000$ & 24 & n.a. & speed movement & 25 & n.a. & n.a. & n.a. & yes & NSW & n.a. & n.a. & n.a. & n.a. & n.a. & n.a. & n.a. & $\begin{array}{l}\mathrm{LC}_{50}\left(\mathrm{~K}_{2} \mathrm{Cr}_{\mathrm{r}_{2}} \mathrm{O}_{7}\right)=32 \mathrm{mg} / \mathrm{L} \\
\mathrm{L} \mathrm{L}_{0}\left(\mathrm{C}\left(\mathrm{N}\left(\mathrm{NO}_{3}\right)_{2}\right)=626 \mathrm{mg} / \mathrm{L}\right. \\
\text { (speed movement) }\end{array}$ \\
\hline $\begin{array}{l}\text { Larsen et al. } \\
(2008)\end{array}$ & $\mathrm{s}$ & $\begin{array}{l}\text { Temperature, } \\
\text { viscosity }\end{array}$ & $\begin{array}{l}T=10-15-20^{\circ} \mathrm{C}, \\
\text { viscosity } \\
1.1-1.2-1.4-1.6 \\
\left(\times 10-6 \mathrm{~m}^{2} \mathrm{~s}^{-1}\right)\end{array}$ & n.a. & n.a. & SW velocity & n.a. & n.a. & n.a. & n.a. & n.a. & NWS & $\begin{array}{l}\text { Rhodomon } \\
\text { sp. }\end{array}$ & nas.a. & n.a. & glass flasks & n.a. & n.a. & n.a. & $\begin{array}{l}\text { Inhibition of } S W \text { velocity for a } \\
10^{\circ} \mathrm{C} \text { temperature reduction }\end{array}$ \\
\hline $\begin{array}{l}\text { Manfra et al. } \\
(2015 \mathrm{~b})\end{array}$ & s & $\begin{array}{l}\text { 1. } \mathrm{CuSO}_{4} ; 2 . \\
\mathrm{SDS} ; 3 . \mathrm{DEG}\end{array}$ & $\begin{array}{l}\text { 1. 2-4-8-16-32; } 2 . \\
3-6-12-24-48 ; 3 . \\
10000-20000- \\
40000-80000- \\
160000\end{array}$ & 48 & 20 & SW and $M$ & $25 \pm 1$ & $35 \pm 1$ & n.a. & D & n.a. & ASW & n.a. & 10 & 3 & MP(24) & 1 & $\begin{array}{l}\text { control } \\
\text { mortal- } \\
\text { ity } \leq 10 \%\end{array}$ & $\begin{array}{l}1 . \\
\mathrm{CuSO}_{4} \\
2 . \mathrm{SDS}\end{array}$ & $\begin{array}{l}\text { 1. } \mathrm{LC}_{50}(24 \mathrm{~h})=14.21 \pm 10.63 \\
\mathrm{LC}_{50}(48 \mathrm{~h})=2.51 \pm 0.22 ; 1 \\
\mathrm{EC}_{50}(24 \mathrm{~h})=5.03 \pm 0.58 \\
\mathrm{EC}_{50}(48 \mathrm{~h})=2.51 \pm 0.37 ; 2 \\
\mathrm{LC}_{50}(24 \mathrm{~h})=19.41 \pm 1.00 \\
\mathrm{LC} \mathrm{C}_{50}(48 \mathrm{~h})=15.60 \pm 0.27 ; 2 \\
\mathrm{EC}_{50}(24 \mathrm{~h})=16.15 \pm 0.32 ; \\
\mathrm{EC}_{50}(48 \mathrm{~h})=7.48 \pm \pm 1.33 ; 3 \\
\mathrm{LC} \mathrm{C}_{50}(24 \mathrm{~h})>160000 \\
\mathrm{LC} \mathrm{C}_{50}(48 \mathrm{~h})>160000 ; 3 \\
\mathrm{EC}_{50}(24 \mathrm{~h})=80370 \pm 3950 ; \\
\mathrm{EC}_{50}(48 \mathrm{~h})=64560 \pm 3420\end{array}$ \\
\hline $\begin{array}{l}\text { Venkateswara } \\
\text { Rao et al. } \\
\text { (2007) }\end{array}$ & s & $\begin{array}{l}\text { 1. CPP; 2. PF; } 3 . \\
\text { MCP; } 4 \text {. ACEP }\end{array}$ & $\begin{array}{l}1.0 .385 \pm 0.08 ; 2 . \\
7.71 \pm 1.48 ; 3 \\
262.68 \pm 17.30 ; 4 \\
2350.07 \pm 131\end{array}$ & 24 & $\begin{array}{l}1000 \\
\text { nauplii }\end{array}$ & $\begin{array}{l}\text { SW speed }(\mathrm{cm} / \mathrm{s}) \\
\text { and distance } \\
\text { travelled ( } \mathrm{m} / \mathrm{min})\end{array}$ & n.a. & n.a. & n.a. & n.a. & n.a. & ASW & n.a. & $\begin{array}{l}\text { 25/each } \\
\text { expo- } \\
\text { sure }\end{array}$ & n.a. & MP(96) & n.a. & n.a. & n.a. & $\begin{array}{l}\text { SW speed inhibition (\%): } \\
1.89 .00 \pm 3.32 \text { CPP } 2 . \\
74.68 \pm 2.13 \mathrm{PF} 3.62 .48 \pm 1.87 \\
\text { MCP 4. 49.83 } 1.53 \text { ACEP }\end{array}$ \\
\hline $\begin{array}{r}\text { Williams } \\
(1994 \mathrm{a}, \\
1994 \mathrm{~b})\end{array}$ & s & No toxicant & n.a. & n.a. & n.a. & $\begin{array}{l}\text { 1. newly hatched } \\
\text { larval (nauplii); } 2 \text {. } \\
\text { larvae with visible } \\
\text { limb buds; } 3 \text {. a series } \\
\text { of larval stages } \\
\text { identified by the } \\
\text { number of actively } \\
\text { beating trunk } \\
\text { appendages present }\end{array}$ & 21 & $35-40$ & n.a. & n.a. & yes & $\begin{array}{l}\text { Brine } \\
\text { solu- } \\
\text { tion }\end{array}$ & $\begin{array}{l}\text { Yeast } \\
\text { and } \\
\text { green } \\
\text { algae }\end{array}$ & n.a. & n.a. & n.a. & n.a. & n.a. & n.a. & $\begin{array}{l}\text { Initial development of new } \\
\text { limbs in Artemia larvae is } \\
\text { unimportant for propulsion. }\end{array}$ \\
\hline
\end{tabular}

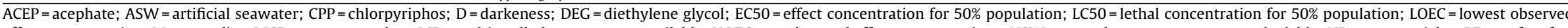

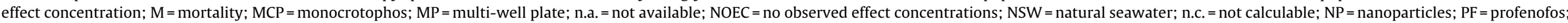
$\mathrm{QC}=$ quality negative control; $\mathrm{S}=$ static; $\mathrm{SDS}=$ sodium dodecylsulphate; $\mathrm{SW}=$ swimming. 
Table 2

Artemia spp. short-term toxicity tests.

\begin{tabular}{|c|c|c|c|c|c|c|c|c|c|c|c|c|c|c|c|c|c|c|c|c|}
\hline References & Test & Toxicant & $\begin{array}{l}\text { Concentration } \\
(\mathrm{mg} / \mathrm{L})\end{array}$ & $\begin{array}{l}\text { Exposure } \\
\text { (h) }\end{array}$ & $\begin{array}{l}\text { Cysts } \\
\text { (mg) }\end{array}$ & Endpoint & $\mathrm{T}\left({ }^{\circ} \mathrm{C}\right)$ & $\begin{array}{l}\text { Salinity } \\
(\%)\end{array}$ & $\mathrm{pH}$ & $\begin{array}{l}\text { Test light } \\
(\mathrm{lx})\end{array}$ & Aeration & $\begin{array}{l}\text { Dilution } \\
\text { medium }\end{array}$ & $\begin{array}{l}\text { Food } \\
\text { (cell/mL) }\end{array}$ & $\begin{array}{l}\text { Organisms } \\
\text { per } \\
\text { chamber }\end{array}$ & Replicates & $\begin{array}{l}\text { Test } \\
\text { vessel } \\
\text { (mL) }\end{array}$ & $\begin{array}{l}\text { Test } \mathrm{mL} \\
\end{array}$ & QC & $\begin{array}{l}\text { Reference } \\
\text { toxicant }\end{array}$ & $\mathrm{NOEC} / \mathrm{LOEC} / \mathrm{LC}_{50} / \mathrm{EC}_{50}$ \\
\hline $\begin{array}{l}\text { APAT and IRSA-CNR } \\
\text { (2003) }\end{array}$ & S, A & $\begin{array}{l}\mathrm{SDS}, \\
\mathrm{K}_{2} \mathrm{Cr}_{2} \mathrm{O}_{7} \\
\mathrm{CuSO}_{4}\end{array}$ & $\begin{array}{l}\text { SDS: } \\
\text { 5-9-16-28-50; } \\
\mathrm{K}_{2} \mathrm{Cr}_{2} \mathrm{O}_{7}: \\
5-9-16-28-50 ; \\
\mathrm{CuSO}_{4}: 1.5- \\
2.7-4.8-8.4-15\end{array}$ & 24 & n.a. & $\mathrm{M}$ & $25 \pm 2$ & n.a. & $6.5-8.5$ & D & n.a. & ASPM, IO & n.a. & 10 & 3 & $\begin{array}{l}\text { MP } 24 \\
\text { wells }\end{array}$ & 1 & $\mathrm{CM} \leq 20 \%$ & $\begin{array}{l}\text { SDS, } \\
\mathrm{K}_{2} \mathrm{Cr}_{2} \mathrm{O}_{7}, \\
\mathrm{CuSO}_{4}\end{array}$ & $\begin{array}{l}\text { Probit analysis, } \\
\mathrm{Chi}^{2} \text { test: } \\
\mathrm{EC}_{50}(\mathrm{SDS}) \\
=23.2 \pm 6.5 \mathrm{mg} / \mathrm{L} \\
\text { with ASPM and } \\
25.6 \pm 5.5 \mathrm{mg} / \mathrm{L} \\
\text { with } \mathrm{IO} \text {. } \\
\mathrm{EC}_{50}\left(\mathrm{~K}_{2} \mathrm{Cr}_{2} \mathrm{O}_{7}\right) \\
=16 \pm 8.4 \mathrm{mg} / \mathrm{L} \text { with } \\
\mathrm{ASPM} \text { and } \\
15.1 \pm 9.6 \mathrm{mg} / \mathrm{L} \\
\text { with } \mathrm{IO} \text {. } \\
\mathrm{EC} 50 \text { (CuSO } \\
=4.5 \pm 2.0 \mathrm{mg} / \mathrm{L} \\
\mathrm{ASPM} \text { and } \\
4.5 \pm 2.0 \mathrm{mg} / \mathrm{L} \text { with } \\
\mathrm{IO}\end{array}$ \\
\hline Artoxkit (2014) & $\mathrm{S}, \mathrm{A}$ & $\mathrm{K}_{2} \mathrm{Cr}_{2} \mathrm{O}_{7}$ & $\begin{array}{l}10-18-32-56- \\
100\end{array}$ & 24 & n.a. & M & 25 & 35 & n.a. & D & n.a. & ASW & no & 10 & 3 & $\begin{array}{l}\text { MP } 24 \\
\text { wells }\end{array}$ & 1 & $\mathrm{CM} \leq 10 \%$ & $\mathrm{~K}_{2} \mathrm{Cr}_{2} \mathrm{O}_{7}$ & n.a. \\
\hline Brix et al. (2004) & $\mathrm{S}, \mathrm{A}$ & $\mathrm{SeO}_{4}{ }^{2-}$ & $\begin{array}{l}42-56-75- \\
100,-133\end{array}$ & 95 & n.a. & M & $25 \pm 1$ & 80-102 & 7.9-8.4 & n.a. & n.a. & NSW & $\begin{array}{l}\text { daily } \\
2 \mathrm{~mL} \text { of } \\
5 \times 10^{5} \\
\text { cell/mL } \\
\text { Platy- } \\
\text { monas } \\
\text { sp. }\end{array}$ & n.a. & 4 & Beakers & 400 & n.a. & n.a. & $\begin{array}{l}\mathrm{LC}_{50}=78(71-86) \\
\mathrm{mg} / \mathrm{L}\end{array}$ \\
\hline Guzzella (1997) & $\mathrm{S}, \mathrm{A}$ & $\begin{array}{l}\text { SDS, } \\
\mathrm{K}_{2} \mathrm{Cr}_{2} \mathrm{O}_{7}, \\
\mathrm{CuSO}_{4}\end{array}$ & $\begin{array}{l}\text { SDS: 5-9-16- } \\
28-50 \mathrm{~K}_{2} \mathrm{Cr}_{2} \mathrm{O}_{7}: \\
5-9-16-28-50 \\
\mathrm{CuSO}_{4}: 1.5- \\
2.7-4.8-8.4-15\end{array}$ & 24 & n.a. & M & $25 \pm 2$ & n.a. & $6.5-8.5$ & D & n.a. & ASPM, IO & n.a. & 10 & 3 & $\begin{array}{l}\text { MP } 24 \\
\text { wells }\end{array}$ & 1 & $\mathrm{CM} \leq 20 \%$ & $\begin{array}{l}\mathrm{SDS}, \\
\mathrm{K}_{2} \mathrm{Cr}_{2} \mathrm{O}_{7}, \\
\mathrm{CuSO}_{4}\end{array}$ & $\begin{array}{l}\text { Probit analysis, } \\
\text { Chi }{ }^{2} \text { test: } \\
\mathrm{EC}_{50}(\mathrm{SDS}) \\
=23.2 \pm 6.5 \mathrm{mg} / \mathrm{L} \\
\text { with ASPM and } \\
25.6 \pm 5.5 \mathrm{mg} / \mathrm{L} \\
\text { with } \mathrm{IO} \text {. } \\
\mathrm{EC}_{50}\left(\mathrm{~K}_{2} \mathrm{Cr}_{2} \mathrm{O}_{7}\right) \\
=16 \pm 8.4 \mathrm{mg} / \mathrm{L} \text { with } \\
\text { ASPM and } \\
15.1 \pm 9.6 \mathrm{mg} / \mathrm{L} \\
\text { with } \mathrm{O} \text {. } \\
\mathrm{EC} \mathrm{C}_{50}\left(\mathrm{CuSO} \mathrm{S}_{4}\right) \\
=4.5 \pm 2.0 \mathrm{mg} / \mathrm{L} \\
\mathrm{ASPM} \text { and } \\
4.5 \pm 2.0 \mathrm{mg} / \mathrm{L} \text { with } \\
\mathrm{IO}\end{array}$ \\
\hline Kissa et al. (1984) & $\mathrm{S}, \mathrm{A}$ & $\begin{array}{l}\text { 1. } \mathrm{CdCl}_{2} \\
\text { 2. } \\
\mathrm{Na}_{2} \mathrm{CrO}_{4} \\
\text {.. } \\
\mathrm{Ni}\left(\mathrm{NO}_{3}\right)_{2} \\
\text { 4. } \\
\mathrm{Co}\left(\mathrm{NO}_{3}\right)_{3}\end{array}$ & $\begin{array}{l}1,2,30-200 \\
4.0-250\end{array}$ & 24,48 & n.a. & M & $24 \pm 0.5$ & n.a. & n.a. & n.a. & n.a. & NSW & n.a. & $\begin{array}{l}20 \text { (3 } \\
\text { days old })\end{array}$ & n.a. & $\begin{array}{l}\text { Glass } \\
\text { cuppels }\end{array}$ & 50 & n.a. & n.a. & $\begin{array}{l}\mathrm{L} C_{50}(48 \mathrm{~h}): \\
160 \mathrm{mg} / \mathrm{L} \text { for Cd; } \\
163 \mathrm{mg} / \mathrm{L} \text { for Ni; } \\
172 \mathrm{mg} / \mathrm{L} \text { for Co; } \\
8 \mathrm{mg} / \mathrm{L} \text { for Cr }\end{array}$ \\
\hline $\begin{array}{l}\text { Libralato et al. } \\
\text { (2007) }\end{array}$ & $\mathrm{S}, \mathrm{A}$ & $\begin{array}{l}\text { Wood } \\
\text { leachates }\end{array}$ & $\begin{array}{l}12-25-50- \\
100 \% \\
\mathrm{v} / \mathrm{v}\end{array}$ & 24 & n.a. & M & 25 & 35 & $7.0-8.3$ & D & n.a. & IO & n.a. & $10-15$ & 3 & $\begin{array}{l}\text { MP } 24 \\
\text { wells }\end{array}$ & 2 & $\mathrm{CM} \leq 10 \%$ & $\mathrm{CuSO}_{4}$ & $\begin{array}{l}\text { Trimmed } \\
\text { Spearman-Karber } \\
\text { expressed as } \mathrm{LC}_{50} \\
\text { and transformed } \\
\text { into Toxicity Units } \\
\left(\mathrm{TU}_{50}\right): 47.57 \\
(41.65-54.75) \text { for } \\
\text { Quercus spp. and } \\
78.21(75.58-84.09) \\
\text { for Picea abies }\end{array}$ \\
\hline
\end{tabular}


Table 2 (Continued)

\begin{tabular}{|c|c|c|c|c|c|c|c|c|c|c|c|c|c|c|c|c|c|c|c|c|}
\hline References & Test & Toxicant & $\begin{array}{l}\text { Concentration } \\
(\mathrm{mg} / \mathrm{L})\end{array}$ & $\begin{array}{l}\text { Exposure } \\
\text { (h) }\end{array}$ & $\begin{array}{l}\text { Cysts } \\
(\mathrm{mg})\end{array}$ & Endpoint & $\mathrm{T}\left({ }^{\circ} \mathrm{C}\right)$ & $\begin{array}{l}\text { Salinity } \\
(\%)\end{array}$ & $\mathrm{pH}$ & $\begin{array}{l}\text { Test light } \\
\text { (lx) }\end{array}$ & Aeration & $\begin{array}{l}\text { Dilution } \\
\text { medium }\end{array}$ & $\begin{array}{l}\text { Food } \\
\text { (cell/mL) }\end{array}$ & $\begin{array}{l}\text { Organisms } \\
\text { per } \\
\text { chamber }\end{array}$ & Replicates & $\begin{array}{l}\text { Test } \\
\text { vessel } \\
(\mathrm{mL})\end{array}$ & Test $\mathrm{mL}$ & QC & $\begin{array}{l}\text { Reference } \\
\text { toxicant }\end{array}$ & $\mathrm{NOEC} / \mathrm{LOEC} / \mathrm{LC}_{50} / \mathrm{EC}_{50}$ \\
\hline $\begin{array}{c}\text { Manfra et al. } \\
(2015 \mathrm{a}, \mathrm{b})\end{array}$ & S, A & $\mathrm{CuSO}_{4}$ & n.a. & 24 & n.a. & M & $25 \pm 1$ & $35 \pm 1$ & n.a. & n.a. & n.a. & ASW & n.a. & 10 & 3 & $\begin{array}{l}\text { MP 24 } \\
\text { wells }\end{array}$ & 1 & $\mathrm{CM} \leq 10 \%$ & $\mathrm{CuSO}_{4}$ & $\begin{array}{l}\text { Trimmed } \\
\text { Spearman-Karber: } \\
\text { mean } \mathrm{EC}_{50} \\
\text { between } 5.63 \text { and } \\
23.31 \mathrm{mg} / \mathrm{L} \\
(\mathrm{CV}<40 \%)\end{array}$ \\
\hline $\begin{array}{l}\text { Persoone et al. } \\
\text { (1993) }\end{array}$ & S, A & $\mathrm{CuSO}_{4}$ & n.a. & 24 & n.a. & M & n.a. & n.a. & n.a. & n.a. & n.a. & ASW & n.a. & 10 & n.a. & $\begin{array}{l}\text { MP } 24 \\
\text { wells }\end{array}$ & n.a. & n.a. & $\mathrm{CuSO}_{4}$ & $\begin{array}{l}\text { Mean LC50 } \\
\left(\mathrm{Cu}^{2+} \mathrm{mg} / \mathrm{L}\right) \text { : from } \\
\text { 3.3 to 3.8; CV\%: } \\
\text { from 32.8 to } 50.7\end{array}$ \\
\hline $\begin{array}{l}\text { Peteros and Uy } \\
(2010)\end{array}$ & S, A & $\begin{array}{l}\text { crude } \\
\text { extracts } \\
\text { of four } \\
\text { medicinal } \\
\text { plants }\end{array}$ & 10-100-1000 & 24 & n.a. & M & n.a. & n.a. & n.a. & L & n.a. & NSW & n.a. & 10 & 3 & Vials & 5 & n.a. & DMSO & 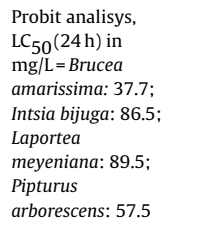 \\
\hline Solis et al. (1993) & S, A & $\begin{array}{l}21 \text { active } \\
\text { agents } \\
\text { from } \\
\text { plants }\end{array}$ & n.a. & 24 & n.a. & M & $22-29$ & n.a. & n.a. & D & n.a. & NSW & n.a. & n.a. & 3 & $\begin{array}{l}\text { MP } 96 \\
\text { wells }\end{array}$ & 0.1 & n.a. & DMSO & $\begin{array}{l}\text { Probit analysis } \\
\text { mean } \mathrm{LC}_{50} \text { value: } \\
\text { between } \\
3.8 \times 10^{-4} \pm 1.4 \times 10^{-4} \\
\text { and }>1000 \mathrm{mg} / \mathrm{L}\end{array}$ \\
\hline Toğulga (1998) & S, A & $\begin{array}{l}\text { SDS, } \\
\mathrm{K}_{2} \mathrm{Cr}_{2} \mathrm{O}_{7}\end{array}$ & n.a. & 24 & n.a. & M & $25 \pm 1$ & n.a. & $7.5 \pm 0.5$ & D & yes & NSW & no & n.a. & n.a. & Petri dish & 10 & n.a. & & $\begin{array}{l}\text { Litchfield and } \\
\text { Wilcoxon' method, } \\
\text { LC } C_{50}: 14.5 \mathrm{SDS} \\
\mathrm{mg} / \mathrm{L} ; 34 \mathrm{PDK} \mathrm{mg} / \mathrm{L} \text {. } \\
\text { Bliss' method LC } 50 \\
=13.8 \pm 0.005 \mathrm{SDS} \\
\mathrm{mg} / \mathrm{L} ; \mathrm{LC}_{50} \\
=32.84 \pm 0.007 \mathrm{PDK} \\
\mathrm{mg} / \mathrm{L}\end{array}$ \\
\hline $\begin{array}{l}\text { Vanhaecke et al. } \\
\text { (1980) }\end{array}$ & S, A & SDS & n.a. & 24 & 100 & M & $25 \pm 1$ & 35 & 7.5 & D & n.a. & $\begin{array}{l}\text { Kinne } \\
\text { (1971) }\end{array}$ & no & 10 & 8 & Petri dish & 10 & n.a. & SDS & $\begin{array}{l}\text { Litchfield and } \\
\text { Wilcoxon: Instar I } \\
\text { mean LC } \\
=33.9 \pm 5.2 \mathrm{mg} / \mathrm{L} \\
\text { (CV }=7.7 \%) ; \text { Instar } \\
\text { IIIIIII mean LC } \mathrm{C}_{50} \\
=17.8 \pm 0.9 \mathrm{mg} / \mathrm{L} \\
(\mathrm{CV}=5.1 \%)\end{array}$ \\
\hline $\begin{array}{l}\text { Vanhaecke et al. } \\
\text { (1981) }\end{array}$ & S, A & SDS & n.a. & 24 & 100 & M & $25 \pm 1$ & $35 \pm 1$ & $8.0 \pm 0.5$ & D & Continuous & IO & n.a. & 10 & 3 & $\begin{array}{l}\text { Glass } \\
\text { Petri dish }\end{array}$ & 10 & $\mathrm{CM} \leq 10 \%$ & SDS & $\begin{array}{l}\text { Litchfield and } \\
\text { Wilcoxon } \\
13.3<\mathrm{LC} C_{50}(24 \mathrm{~h}- \\
\text { SDS }<19.9 \mathrm{mg} / \mathrm{L}\end{array}$ \\
\hline
\end{tabular}

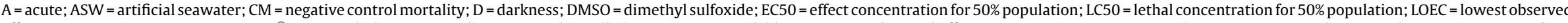

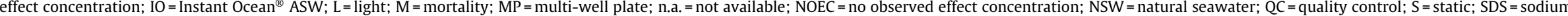
dodecylsulphate. 
Methanol and ethanol toxicity was evaluated (Vismara, 1998) by exposing hydrated cysts for $48 \mathrm{~h}$. The hatching test was also successfully used to evaluate the toxicity of two biocides utilized in antifouling paints (Diuron and Irgarol) (Alyürüc and Çavas, 2013) by exposing hydrated cysts for $24 \mathrm{~h}$. The hatching test was also able to evaluate, in a concentration-dependent way, the toxicity of water soluble algal extracts and crude cellular extracts of Skeletonema costatum and Nitzschia commutata, and the diatom-derived short chain aldehydes decadienal by exposing 20-30 cysts per replicate to fresh seawater $(1 \mathrm{~mL})$ at $15^{\circ} \mathrm{C}$ in multiplate wells (Caldwell et al., 2003).

A quite different protocol using commercial brine shrimp hatcher was applied to evaluate the toxicity of three antibiotics (Bacitracin, Flumequine and Sulfadimethoxine) (Migliore et al., 1993a, 1997), two organic compounds (diethylene glycol (DEG) and sodium dodecyl sulphate (SDS)) (Rotini et al., 2015), and the bioactivity of extracts of marine invertebrates and macroalgae (isopropanolic extracts from Hyatella spp. (sponge), Dysidea spp. (sponge), Pacifigorgia adamsii (coral) and Muricea sp. (coral) already found active against two human cell lines) (Carballo et al., 2002). This method is aimed to evaluate the dry biomass of hatched nauplii from $1 \mathrm{~g}$ ( $\sim 200,000$ individuals) of Artemia spp. cysts incubated at $25^{\circ} \mathrm{C}$ and 35 PSU under constant illumination (3000 lx) and aeration (air bubbling, not compulsory) for 2-4 days. Cysts were intact and not dechorionated. The free nauplii, after the successful emergence from the cyst and hatching, aggregate over a sieve in the center of the hatcher due to their positive phototactic activity easing their collection. This kind of approach seems to be more viable for the evaluation of the reduced emergence and/or hatching than enumerating one-by-one the cysts able to complete their embryonic development when exposed to a toxicant (Kuwabara et al., 1980; Rotini et al., 2015). Phototaxis is a cost-effective and easy way of nauplii grouping for counting or biomass production determination. Anyhow, this method does not allow discriminating the sub-lethal etiology of the treatment exposure. Further investigation would be interesting about Artemia spp. light reception, nervous system processing, motor function as well as developmental monitoring.

Another group of 'cyst based tests' evaluated the morphological disorders and size of hatched nauplii (Rafiee et al., 1986; Vismara, 1998; Neumeyer et al., 2014). Bagshaw et al. (1986) found reversible arrest of development of pre-nauplii larvae emerged in $\mathrm{Cd}$ spiked waters $\left(10 \mu \mathrm{M} \mathrm{CdCl}_{2}\right)$ and morphological alteration at higher concentrations, considering the phenotypic alterations an endpoint within the hatching test. Also in the case of $\mathrm{Hg}$, Go et al. (1990) described the presence of morphological abnormalities and delayed development in exposed cysts during emergence and hatching phase.

Rafiee et al. (1986) incubated hydrated cysts up to $100 \mathrm{~h}$ in presence of $\mathrm{Cd}$ and $\mathrm{Zn}$ spiked seawater. Scanning electron micrograph and light microscopy evidenced morphological abnormalities and delayed development. Migliore et al. (1997) according to Rafiee et al. (1986) highlighted severe phenotypic alteration due to Bacitracin and changes in pigmentation due to Flumequine. Neumeyer et al. (2014) investigated the use of fully hydrated and dechorionated embryos (15-28 per replicate) incubated at $21-23^{\circ} \mathrm{C}$ and room light condition observing and describing by light microscopy all Artemia development phases and relative alterations considering a range of salinity $(20,25,30$ and $35 \%$ ) and density (from 1-3 embryo/mL to approximately 350 embryo $/ \mathrm{mL}$ ). They found that (i) aberrant morphologies result from a mismatch between development and emergence; (ii) a small salinity increase or embryo abundance decrease can impair the emergence success having only moderate effects on the development; (iii) aberrant developmental paths produce swimming nauplii and unhatched cysts appearing normal in counts. Neumeyer et al. (2014) improved the design of Artemia spp, endpoint assay offering an alternative approach considering the quantitative developmental profile under specified conditions $\left(22^{\circ} \mathrm{C}, 16: 8\right.$ or $0: 24$ light:dark cycle, $20-30 \%$ artificial seawater), even if it can be a quite time consuming activity.

Some authors compared the sensitivity of hatching and acute/chronic mortality toxicity test. Jorgensen and Jensen (1977) observed that when Artemia salina is exposed to $\mathrm{Cu}$ ions the hatching rate is much more susceptible than mortality, obtaining $\mathrm{EC}_{50}$ values at least 100 times below the analogous $\mathrm{LC}_{50}$. Migliore et al. (1997) showed that in A. franciscana both cysts hatching and mortality responded to antibiotics exposure evidencing hatching rate and larvae morphological alterations, and nauplii death, respectively. Carballo et al. (2002) evidenced a similar sensitivity between cysts' hatchability and nauplii mortality exposed to various organic extracts, suggesting a simultaneous application of both assays to test natural marine products' pharmacological activity. Sarabia et al. (2003) conducted experiments by exposing cysts and larvae to $\mathrm{Cd}$. They did not observe toxic effects on cysts (percentage and timing of cyst hatching) and larvae (life span and reproductive parameters). Caldwell et al. (2003) compared hatching success and larval mortality of $A$. salina exposed to diatom extracts and aldehydes, showing that hatchability is a less sensitive endpoint. The most recent paper by Rotini et al. (2015) compared the results of hatching, acute mortality and chronic mortality tests on DEG and SDS ranking the endpoints as follows: acute mortality < hatchability < chronic mortality. The protocol described in Rotini et al. (2015) was intercalibrated at Italian level between 2006-2009 involving eleven laboratories to ring toxicity tests with A. franciscana using $\mathrm{CuSO}_{4}$ as reference toxicant (Manfra et al., 2015b).

\subsubsection{Biomarkers}

Biomarkers are any measurable characteristics of an organism reflecting a specific or general physiological and health state. Biomarkers are often compounds isolated from serum, hemocyanin, or other fluids that can be used as an indicator of the presence or severity of a particular disease state. The brine shrimp A. salina was used in few screenings considering biochemical mechanisms (Nunes et al., 2006b). Espiritu et al. (1995) developed a $1 \mathrm{~h}$ enzymatic inhibition assay (Fluotox) with Artemia nauplii using a fluorigenic enzyme substrate comparing the output with 24 and $48 \mathrm{~h}$ mortality test. Addition of 4 -methylumbelliferyl- $\beta$-Dgalactoside (MUF) to the test medium did not influence the toxicity of $\mathrm{CuSO}_{4}, \mathrm{CdCl}_{2}, \mathrm{~K}_{2} \mathrm{Cr}_{2} \mathrm{O}_{7}$, sodium pentachlorophenate, SDS, and phenol. Uptake of MUF and its subsequent hydrolysis form the 4-methylumbelliferone that is strongly fluorescent in alkaline solution using a UV light. The exposure to toxicants may reduce or inhibit this enzymatic pathway that can be used as an early manifestation of toxicity.

Varó et al. (2002) observed the inhibitory potential of chlorpyrifos and dichlorvos on acetylcholinesterase (AChE) activity using A. salina and $A$. parthenogenetica provoking the accumulation of acetylcholine (ChE) at neuromuscular junction disrupting the function nerve (Peakall, 1992). Results highlighted the tolerance of $A$. salina to high levels of ChE inhibition (approximately 80\%) without lethal effects.

Few data exist on the antioxidant defense mechanisms in Artemia spp. Nunes et al. (2006b) investigated the antioxidant skills of $A$. parthenogenetica to pharmaceuticals and personal care products. Benzodiazepine significantly inhibited ChE and glutathione-peroxidase (GPx) activities. Clofibrate and clofibric acid showed significant decreases in Se-dependent GPX. In particular, clofibrate caused a slight increase of lipid peroxidation (LP) (thiobarbituric acid reactive substances, TBARS) (Nunes et al., 2006b). Sodium dodecylsulphate (SDS) widely used in detergents 
decreased the activity of both $\mathrm{ChE}$ and glutathione reductase (GRed) in A. parthenogenetica (Nunes et al., 2006b).

Nauplii of $A$. franciscana were selected as model organisms to assess the induction of acute oxidative stress of Aloe vera juice (Sirdaarta and Cock, 2008, 2010) showing 34\%, 79\% and $90 \%$ inhibition of thioredoxin reductase (TRed), GRed and GPX, respectively. Thus, an oxidative stress was induced after acute exposure (Sirdaarta and Cock, 2008, 2010) observing that vitamin E could help to partially reduce the effects of $A$. vera juice exposure (Sirdaarta and Cock, 2010).

Glutathione $S$-transferase (GST) activity was investigated during the early developmental stages of Artemia spp. hatching in artificial seawater prepared with municipal wastewater effluent. Alterations of the isoenzyme profile were evidenced with a maximum effect after $48 \mathrm{~h}$ from hatching. The isoenzyme profile depended upon the organism age and can be affected by environmental factors such as water quality (Grammou et al., 2011).

Katranitsas et al. (2003) measured adenylpyrophosphatase (ATPase) and aldehyde dehydrogenase (ALDH) in brine shrimps exposed to $\mathrm{Cu}$-based antifouling paints evidencing an alteration of Artemia osmoregulation up to death. Also Castritsi-Catharios et al. (2012) suggested that ALDH activity in Artemia nauplii could be a valuable biomarker for the evaluation of antifouling paint.

The heat stress proteins (HSPs) in Artemia were observed in early developmental stages, in encysted gastrula embryos and nauplii. HSP26 is undetectable in unstressed cells, appearing in presence of heat or salt shock, cell cycle arrest, nitrogen or carbon starvation, oxidative stress and low pH. The protein p26 is a small heat shock $/ \alpha$-crystalline protein exhibiting an in vitro molecular chaperone activity conferring a thermo-tolerance (Thomas, 2003). Its synthesis plays a critical role in embryo encystment, diapause and quiescence (Delinger, 2002; Miah et al., 2010).

Proteomic approaches were considered to compare nonstressed (negative control) and stressed organisms (Migliore et al., 2007) improving the understanding of physiological mechanisms underlying stress response and/or tolerance and highlighting putative biomarkers in the differentially expressed proteins (Rotini et al., 2013). For example, the microtubule proteome encompasses tubulin and a group of proteins associated with tubulin upon microtubule formation determining microtubule organization and functioning. In post-diapause development $(0$ and $12 \mathrm{~h})$ cell free extracts of $A$. franciscana, tubulin assembly was investigated in presence and absence of taxol (Paclitaxel, $10 \mu \mathrm{M}$ ) (O'Connell et al., 2006) evidencing poorly formed microtubules and abundant low molecular mass proteins (Day et al., 2003). No proteins were present in the absence of taxol. With taxol, ten of the fifty-five proteins identified in A. franciscana proteome appeared at both exposure times ( 0 and $12 \mathrm{~h}$ ): all decreased except for one. Fructose 1,6-bisphosphate aldolase, enolase, HSPs and ATP-binding protein are represented in the undeveloped cysts as isoforms differing in both isoelectric point and molecular mass. In post-diapause A. franciscana, these proteins were present in reduced amount. Because only the 40S ribosomal protein S12 increased within the microtubule proteome during development $(12 \mathrm{~h})$ further in vitro analyses are required for physiological verification.

\subsubsection{Teratogenicity test}

Artemia spp. larval stages growth inhibition can be also considered as a teratogen test system based on disturbance of elongation development (relative to controls raised at the same time and conditions) from 24 to $48 \mathrm{~h}$ in animals cultured in medium containing a presumptive teratogen (Olson, 1979; Kerster and Schaeffer, 1983). Even though the protocol is not suited to particulates, substances volatilized from water solution at $25^{\circ} \mathrm{C}$ or waters may have very low concentrations of teratogens such as municipal wastewaters or receiving waters. Growth length inhibition is calculated consider- ing measurements between the well-pigmented eye and the poorly defined end of the tail by centering an animal in the stereomicroscope's field. With the exception of zinc, chemicals in the $\mu \mathrm{g} / \mathrm{L}$ range were not teratogenic, whereas chemicals between 0.25 and $25 \mathrm{mg} / \mathrm{L}$ were teratogenic $(\mathrm{Cd}, \mathrm{Hg}, \mathrm{Pb}, \mathrm{Zn}$, bromoform, $n$-butylphthalate, 1,2-dichloroethane, nitrobenzene, tetrachloroethylene, toluene, 1,2,4-trichlorobenzene and 1,1,3-thrichloroethane). Nevertheless the limited data, Kerster and Schaeffer (1983) stated that the system is not very sensitive.

\subsubsection{Acute larvae behavioural test}

Data about Artemia spp. short-term acute behavioral test are summarized in Table 1. Swimming speed is the most frequently used behavioral endpoint of physiological status for aquatic organisms (Faimali et al., 2006). Locomotion, like swimming, is used as stress indicator in several ecotoxicological studies representing a sensitive measure of toxic stress for a wide range of environmental contaminants (Little and Finger, 1990). In the last ten years, the swimming behavior was studied as a response to invertebrates' organic or inorganic exposure to pollutants including Artemia. There is still a general lack of appropriate biocompatible automation, optoelectronic sensors and algorithms for behavioral data analysis that could represent a way to solve the major shortcomings not yet addressed in ecotoxicology (e.g. toxicity tests to be performed manually and with low-throughput) (Huang et al., 2015). Williams (1994a,b) applied a physical model regarding the appendages of Artemia to empirically determine force coefficients acting regimes relevant to developing larvae. This first analysis imported equations exploring changes in the mechanics of swimming in animals too small for direct measurement. Larvae are interesting because, compared to adults, they can undergo fluctuations in size and shape. Artemia develops from a small nauplius that ratchets along with one pair of oars to a multi-limbed adult gliding continuously through the water. As the animals approach the adult morphology growing and adding limbs along the trunk, their average swimming speed increases and the jerky swimming of hatchlings changes into the smooth gliding of adults. The gradual change in swimming does not correspond only to the trunk limbs increase, but also to energy budget supporting the movement (Williams, 1994b). Davenport and Healy (2006) assessed the relationship between physical parameters (medium salinity, body density, and buoyancy) and swimming in A. franciscana larvae, finding that the horizontal swimming speed was unaffected by salinity and viscosity (i.e. between $8.5-100 \%$ o), but the observed differences in vertical swimming rates are solely due to the relatively constant body density. Venkateswara Rao et al. (2007) studied the covered distance and swimming speed of Artemia nauplii survivors after $24 \mathrm{~h}$ exposure to 4 pesticides (acephate, chlorpyrifos, monocrotophos and profenofos). A computerized video tracking system (Ethovision, Noldus Information Technology, Wageningen, The Netherlands) was used for the automation of behavioral experiments. Twenty-five nauplii were placed individually into a 96 well culture plate and their behavior was recorded. Authors observed that the AChE enzyme activity might be inhibited by pesticides thus acetylcholine accumulated at neuromuscular level altering the locomotion behavior of organism interrupting the coordination between the nervous and muscular junctions. Larsen et al. (2008) analyzed the effect of temperature-dependent viscosity of ambient seawater (20\%) on the swimming velocity of brine shrimps using video-microscope recordings at various temperatures (between $10^{\circ} \mathrm{C}$ and $20^{\circ} \mathrm{C}$ ) and viscosities (between $1 \times 10^{-6} \mathrm{~m}^{2} / \mathrm{s}$ and $1.6 \times 10^{-6} \mathrm{~m}^{2} / \mathrm{s}$ ) (but keeping a constant temperature of $20^{\circ} \mathrm{C}$ ). They suggested that modifications in swimming velocity of Artemia were due to changes in kinematic viscosity. Recently, Garaventa et al. (2010) recorded the swimming speed alteration of Artemia together with mortality, using a video camera 
with a macro-objective for recording the larval swimming paths. Larvae were dark-adapted for 2 min before the video start (i.e. the experimental time required to reach steady speed and uniform spatial distribution) and the images were analyzed using an advanced image processing software to reconstruct the individual paths/tracks. This system provided a suitable tool to detect linear swimming speed of Artemia, since authors obtained a velocity $(3.05 \mathrm{~mm} / \mathrm{s})$ in accordance with other studies using the same organism. Alterations in swimming speed were detected for various compounds (Zn pyrithione, Macrotrol ${ }^{\circledR}$ MT-200, and eserine) $<0.1-5 \%$ of their $\mathrm{LC}_{50}$ values. Gambardella et al. (2014) used the same system investigating the toxicity of metal oxide nanoparticles (MO-NPs) to A. salina, using swimming speed, and enzymatic activity alterations, and mortality as endpoints thus including continuous or periodic observation of single animals in a nonendpoint perspective. Although no mortality was found, MO-NPs decreased larvae swimming speed and biochemical responses, evidencing the sensitivity of non-lethal endpoints. Alyuruk et al. (2013) proposed another video-tracking method assessing whether monitored Artemia are dead or alive observing colors/textures of nauplii. Authors exposed $A$. salina nauplii at various concentrations between 7 and $100 \mathrm{mg} / \mathrm{L}$ of $\mathrm{K}_{2} \mathrm{Cr}_{2} \mathrm{O}_{7}$ and $p$-coumaric acid. Considering results by microscopy observation, the proposed algorithm tracks and counts were robust relative to the number of motile nauplii. This kind of automated analysis was also able to provide additional quantitative data such as curvature of paths, number of stops and immobile time. Manfra et al. (2016) compared the swimming speed alteration to hatching and mortality exposing Artemia to $\mathrm{CuSO}_{4}$, SDS and DEG. The system for measuring the swimming speed proposed by Garaventa et al. (2010) showed that swimming speed was more sensitive than mortality, with sensitivity similar and sometimes higher than cysts' hatchability.

Recently, Huang et al. (2015) proposed a proof-of-concept lab-on-chip platform capable of performing fully programmable time-lapse and video-microscopy of multiple samples for rapid $A$. franciscana ecotoxicity analysis. This method dynamically detected sub-lethal behavioral endpoints, such as changes in speed of movement or distance travelled by each animal, observing how they changed compared to the negative control exposure after $24 \mathrm{~h}$ contact time. Nevertheless these endpoints present promising perspectives compared to Artoxkit (2014), further research activity is strongly required to identify clear contaminant-response relationships.

\subsubsection{Acute mortality test}

Data about Artemia spp. short-term acute immobilization/mortality test are summarized in Table 2. The Artemia suitability for acute toxicity studies is well documented in the scientific literature over the past 80 years (Boone and Baas-Becking, 1931). The first general method for conducting toxicity tests with Artemia spp. can be attributed to Michael et al. (1956). This paper stimulated further research about the test development and definition of more detailed testing procedures. In 1975, this extensive research led to the creation of the Artemia Reference Center (ARC-test) at Ghent University (Belgium) and the release of the first short-term toxicity protocol (i.e. mortality) with brine shrimp larvae (Vanhaecke et al., 1981; Vanhaecke and Persoone, 1981; Vanhaecke and Persoone, 1984). The bioassay is carried out in static conditions for $24 \mathrm{~h}$ at $25 \pm 1^{\circ} \mathrm{C}$ in darkness in covered glass Petri dishes $(60 \times 12 \mathrm{~mm})$. A homogeneous population of Instar II-III nauplii hatched from cysts $(100 \mathrm{mg})$ was exposed to SDS as reference toxicant. If the test meets the quality criteria it is considered as valid: negative controls $\leq 10 \%$, and $13.3 \mathrm{mg} / \mathrm{L}<\mathrm{LC}_{50}$ (SDS) $<19.9 \mathrm{mg} / \mathrm{L}$, dissolved oxygen (DO) concentration after $24 \mathrm{~h}>2 \mathrm{mg} / \mathrm{L}$ in the lowest concentration with $100 \%$ mortality of larvae (Vanhaecke and Persoone, 1981).
An international intercalibration exercise focused on Vanhaecke and Persoone (1981) protocol assessed its reliability, accuracy and precision considering $\mathrm{K}_{2} \mathrm{Cr}_{2} \mathrm{O}_{7}$ and SDS as reference toxicants for intra- and inter-laboratory exercises. The study involved 67 laboratories (59 laboratories in Europe and 8 in US) (Vanhaecke and Persoone, 1984). For SDS, the interlaboratory coefficient of variation (CV) was $24.82 \%$ and $25.25 \%$ for European and US laboratories, in this order. Considering $\mathrm{K}_{2} \mathrm{Cr}_{2} \mathrm{O}_{7}$ as reference toxicant, the $\mathrm{CV}$ was 34.89 and $18.49 \%$ for European and US, respectively (Vanhaecke et al., 1981; Vanhaecke and Persoone, 1984). Results evidenced that the toxicity test was carried out with little difficulties showing satisfactory and reliable results. Persoone and Castritsi-Catharios (1989a,b) used the approach of the Artemia Reference Center (ARC) (University of Gent, Belgium) test to set a standard protocol for routine toxicity testing of antifouling and other potentially hazardous paints. The effectiveness of this technique was assessed by further studies (Castritsi-Catharios et al., 2007, 2013, 2014). This protocol was engineered in a toxkit called Artoxkit (MicroBioTests Inc., Mariakerke (Gent), Belgium) (Artoxkit, 2014) including a batch of dormant eggs (cysts) of A. franciscana to generate "on demand" larvae eliminating the need for continuous culturing. In 1989, international intercalibration exercises involving 129 laboratories between Europe, USA and Canada investigated the reliability of Artoxkit on $\mathrm{CuSO}_{4}$ as reference toxicant. Results were encouraging with only few participants reporting problems. The precision was not striking with a CV equal to $50 \%$ in Europe and USA, and 33\% in Canada. Criticisms raised about the inexperience of some participants and the instability of $\mathrm{CuSO}_{4}$ in seawater determining a decrease in toxicity over the course of few days and creating some misunderstanding about moribund and really dead nauplii (Persoone et al., 1993). Many authors used the Artoxkit since its commercialization ( $>180$ results in Google Scholar searching for "Artoxkit" 18th Jan 2016) like Koutsaftis and Aoyama (2007).

Solis et al. (1993) suggested a modification of the acute toxicity test developing a new $A$. salina microplate cytotoxicity assay requiring a small amount of testing sample $(0.6 \mathrm{mg})$ up to $1 \mathrm{mg} / \mathrm{mL}$ in artificial sea water. The use of 96 -well microplates in $100 \mu \mathrm{L}$ sea water enabled testing a large number of samples.

A detailed procedure to perform acute test with Artemia spp. was reported by Guzzella (1997) and APAT and IRSACNR (2003) using nauplii at II-III Instar stage. They reported the outcome of an interlaboratory study (acute $24 \mathrm{~h}$ mortality) between five Italian laboratories investigating SDS, $\mathrm{K}_{2} \mathrm{Cr}_{2} \mathrm{O}_{7}$ and $\mathrm{CuSO}_{4}$ as reference toxicants. Toxicity tests were carried out using as dilution water both ASPM artificial seawater $\left(\mathrm{NaCl}=26.4 \mathrm{~g}, \mathrm{KCl}=0.84 \mathrm{~g}, \mathrm{CaCl}_{2} \cdot \mathrm{H}_{2} \mathrm{O}=1.67 \mathrm{~g}, \mathrm{MgCl} \cdot \mathrm{H}_{2} \mathrm{O}=4.6 \mathrm{~g}\right.$, $\mathrm{MgSO}_{4} \cdot 7 \mathrm{H}_{2} \mathrm{O}=5.58 \mathrm{~g}, \mathrm{NaHCO}_{3}=0.17 \mathrm{~g}$ and $\mathrm{H}_{3} \mathrm{BO}_{3}=0.03 \mathrm{~g}$ in $1 \mathrm{~L}$ of ultrapure water) and Instant Ocean. The CV was between $28 \%$ and $52 \%$ using ASPM and between 21\% and 64\% using Instant Ocean, suggesting that ASPM is more suitable than Instant Ocean.

Between 2006-2009, the $24 \mathrm{~h}$ mortality test with $A$. franciscana was intercalibrated together hatching assay (Manfra et al., 2015b). Three inter-comparison results constituted the dataset for calculating the $24 \mathrm{~h} \mathrm{LC}_{50}$ mean and the repeatability/reproducibility coefficients on $\mathrm{CuSO}_{4}$. The CV value and the $r$ and $R$ values indicated acceptable inter-laboratory test-reliability similar to ISO (1996). Guzzella (1997) and APAT and IRSA-CNR (2003) methods were used for cysts hatching. Larvae transfer from cyst hatching Petri dish to test chambers was operated minimizing as much as possible the dilution of test medium. Mortality was defined operationally as total absence of movement (i.e. swimming activity or movement of appendices) after mechanical stimulation (i.e. touching the larvae with the tip of a glass Pasteur pipette) for approximately $10 \mathrm{~s}$ of observation. 
Table 3

\begin{tabular}{|c|c|c|c|c|c|c|c|c|c|c|c|c|c|c|c|c|c|c|c|c|}
\hline References & Test & Toxicant & $\begin{array}{l}\text { Concentratior } \\
(\mathrm{m} / \mathrm{L})\end{array}$ & $\begin{array}{l}\text { nExposure } \\
\text { (h) }\end{array}$ & $\begin{array}{l}\text { Cysts } \\
\text { (mg) }\end{array}$ & Endpoint & $\mathrm{T}\left({ }^{\circ} \mathrm{C}\right)$ & $\begin{array}{l}\text { Salinity } \\
(\%)\end{array}$ & $\mathrm{pH}$ & $\begin{array}{l}\text { Test light } \\
\text { (lx) }\end{array}$ & Aeration & $\begin{array}{l}\text { Dilution } \\
\text { medium }\end{array}$ & $\begin{array}{l}\text { Food } \\
\text { (cell/mL) }\end{array}$ & $\begin{array}{l}\text { Organisms } \\
\text { per } \\
\text { chamber }\end{array}$ & Replicates & $\begin{array}{l}\text { Test } \\
\text { vessel } \\
\text { (mL) }\end{array}$ & $\begin{array}{l}\text { Test } \\
\text { mL }\end{array}$ & QC & $\begin{array}{l}\text { Reference } \\
\text { Toxicant }\end{array}$ & $\mathrm{NOEC} / \mathrm{LOEC} / \mathrm{LC}_{50} / \mathrm{EC}_{50}$ \\
\hline $\begin{array}{l}\text { Browne and } \\
\text { Wanigasekera } \\
\text { (2000) }\end{array}$ & n.a. & Salinity & n.a. & 21 & n.a. & n.a. & $15-24-30$ & $\begin{array}{l}60-120- \\
180\end{array}$ & n.a. & $\begin{array}{l}\text { Fluorescent } \\
\text { light }\end{array}$ & no & $\begin{array}{l}\text { ASW } \\
\text { (renewal } \\
\text { every 3 d } \\
\text { (M) d 7 d } \\
\text { (R) }\end{array}$ & $\begin{array}{l}\text { Yeast, and } \\
\text { Dunaliella } \\
\text { sp. }\end{array}$ & $5 / \mathrm{jar}$ & 10 & jars & $\begin{array}{l}100 \\
\text { (M); } \\
250 \\
\text { (R) }\end{array}$ & n.a. & n.a. & 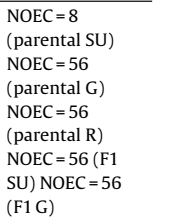 \\
\hline $\begin{array}{l}\text { Cunningham } \\
\text { (1976) }\end{array}$ & n.a. & Dimilin & $\begin{array}{l}0-0.001- \\
0.002- \\
0.005- \\
0.010\end{array}$ & $21-28$ & n.a. & $\mathrm{R}$ & $25 \pm 2$ & 25 & 8.2 & n.a. & n.a. & ASW & $\begin{array}{l}\text { Daily } \\
\text { renewal }\end{array}$ & 15 & 5 & n.a. & n.a. & n.a. & n.a. & n.a. \\
\hline $\begin{array}{l}\text { Nováková } \\
\text { et al. } \\
\text { (2007) }\end{array}$ & n.a. & $\begin{array}{l}\mathrm{CdCl}_{2}, \\
\mathrm{ZnSO}_{4}\end{array}$ & $\begin{array}{l}\text { Cd: 5-10- } \\
\text { 15-25-50- } \\
100-250 ; \\
\text { Zn: 50- } \\
100-250 \mathrm{mg}\end{array}$ & 10 & n.a. & M & $20 \pm 1$ & n.a. & n.a. & n.a. & n.a. & NSW & Glucose & 10 & n.a. & $\begin{array}{l}\text { polystyrene } \\
\text { Petri } \\
\text { dishes }\end{array}$ & 10 & n.a. & n.a. & $\begin{array}{l}\text { Synergistic or } \\
\text { antagonistic } \\
\text { effects }\end{array}$ \\
\hline $\begin{array}{c}\text { Gebhardt } \\
(1976)\end{array}$ & n.a. & $\mathrm{Cd}, \mathrm{Cu}, \mathrm{Hg}$ & $\begin{array}{l}\text { Cd: 0-1.0- } \\
\text { 3.3--33.0; } \\
\text { Cu: 0-0.3- } \\
0.03- \\
0.003 ; \\
\text { Hg: } \\
0-0.001- \\
0.003- \\
0.05\end{array}$ & 25 & n.a. & $\begin{array}{l}\text { G } \\
\text { (length), } \\
\text { R (time } \\
\text { (d) until } \\
\text { nauplii } \\
\text { produc- } \\
\text { tion) }\end{array}$ & $27 \pm 2$ & $150-320$ & n.a. & $\begin{array}{l}16 \mathrm{~h} \text { fluo- } \\
\text { rescent } \\
\text { light }\end{array}$ & n.a. & $\begin{array}{l}\text { NSW } \\
\text { (renewal } \\
\text { every 3 d) }\end{array}$ & $\begin{array}{l}\text { D. viridis } \\
(40-50 \\
\left.\text { cells/mm } / \mathrm{mm}^{3}\right) \\
\text { every 3 d }\end{array}$ & 5 & n.a. & 300 & n.a. & n.a. & n.a. & n.a. \\
\hline $\begin{array}{l}\text { Manfra et al. } \\
\text { (2012) }\end{array}$ & semi-S & SDS & $\begin{array}{l}1.56- \\
3.12- \\
6.25- \\
12.5-25\end{array}$ & 14 & n.a. & M & $25 \pm 2$ & $35 \pm 2$ & n.a. & $\begin{array}{l}900 \pm 100 \\
\text { and } 14 \mathrm{~h} \\
\text { light }\end{array}$ & n.a. & $\mathrm{ASW}^{*}$ & $\begin{array}{l}\text { D. } \\
\text { tertiolecta } \\
\left(1 \times 10^{5}\right. \\
\text { cells } / \mathrm{mL})^{*}\end{array}$ & 10 & 3 & 100 & 50 & $\mathrm{CM} \leq 20 \%$ & SDS & $\mathrm{LC}_{50}=8.0 \pm 5$ \\
\hline $\begin{array}{l}\text { Manfra et al. } \\
\text { (2015a) }\end{array}$ & semi-S & DEG & $\begin{array}{l}12500- \\
25000- \\
50000- \\
100000- \\
200000\end{array}$ & 14 & n.a. & M & $25 \pm 2$ & $35 \pm 2$ & n.a. & $\begin{array}{l}900 \pm 100 \\
\text { with } 14 \mathrm{~h} \\
\text { light }\end{array}$ & n.a. & $\mathrm{ASW}^{*}$ & $\begin{array}{l}D . \\
\text { tertiolecta } \\
\left(1 \times 10^{5}\right. \\
\text { cells } / \mathrm{mL})^{*}\end{array}$ & 10 & 3 & 100 & 50 & $\mathrm{CM} \leq 15 \%$ & n.a. & $\begin{array}{l}\text { NOEC(14 } \\
\text { d) }=25000\end{array}$ \\
\hline $\begin{array}{c}\text { Manfra et al. } \\
(2015 \mathrm{a}, \mathrm{b})\end{array}$ & semi-S & SDS & $\begin{array}{l}1.6-3.1- \\
6.2-12.5- \\
25.0\end{array}$ & 14 & n.a. & M & $25 \pm 2$ & $35 \pm 2$ & n.a. & $\begin{array}{l}900 \pm 100 \\
\text { with } 14 \mathrm{~h} \\
\text { light }\end{array}$ & n.a. & $\mathrm{ASW}^{*}$ & $\begin{array}{l}\text { D. } \\
\text { tertiolecta } \\
\left(1 \times 10^{5}\right. \\
\text { cells } / \mathrm{mL})^{*}\end{array}$ & 10 & 3 & 100 & 50 & $\mathrm{CM} \leq 15 \%$ & $\mathrm{~K}_{2} \mathrm{Cr}_{2} \mathrm{O}_{7}$ & $\begin{array}{l}\mathrm{EC}_{50}(14 \\
\mathrm{d})=8.03 \pm 1.11 \\
(\mathrm{n}=5) ; \mathrm{EC}_{50}(14 \\
\mathrm{d})=8.50 \pm 3.34 \\
(\mathrm{n}=24)\end{array}$ \\
\hline $\begin{array}{l}\text { Savorelli } \\
\text { et al. } \\
(2007)\end{array}$ & semi-S & Dispersant & $\begin{array}{l}3.12- \\
6.25- \\
12.5-25\end{array}$ & $7-14$ & n.a. & $\begin{array}{l}\mathrm{M}, \mathrm{G} \\
\text { (carapace } \\
\text { length) }\end{array}$ & $25 \pm 1$ & $35 \pm 1$ & n.a. & $\begin{array}{l}900 \pm 100 \\
\text { with } 14 \mathrm{~h} \\
\text { light }\end{array}$ & n.a. & $\mathrm{ASW}^{*}$ & $\begin{array}{l}\text { D. } \\
\text { tertiolecta } \\
\left(1 \times 10^{5}\right. \\
\text { cells } / \mathrm{mL})^{*}\end{array}$ & 10 & 3 & 100 & 50 & $\mathrm{CM} \leq 20 \%$ & SDS & $\begin{array}{l}\operatorname{NOEC}(7 \\
\text { d) }) 6.25(\mathrm{M}, \\
\mathrm{G}) ; \operatorname{NOEC}(14 \\
\mathrm{d})=6.25(\mathrm{M} \\
\text { and G, } A \text {. } \\
\text { parthenogenet- } \\
\text { ica); } \mathrm{NOEC}(14 \\
\mathrm{d})=3.12(\mathrm{M}, \mathrm{A} \text {. } \\
\text { franciscana) } \\
\text { NOEC( } \mathrm{d} \text { ) } \\
\text { dispersant }=5 \\
\text { (M, } A \text {. } \\
\text { franciscana) }\end{array}$ \\
\hline
\end{tabular}

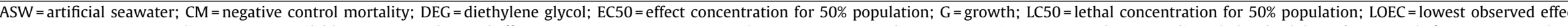
concentrations; $\mathrm{M}=$ Mortality; n.a. = not available; NOEC = no observed effect concentrations; NSW = natural seawater; $\mathrm{R}=$ reproduction; S= static; SU = survival; SDS = sodium dodecyl sulphate; * = renewal after 2-5-7-9-12 d. 


\subsection{Long-term toxicity testing}

Data about Artemia spp. long-term immobilization/mortality test are summarized in Table 3. Most efforts in developing toxicity tests with Artemia spp. have been focused on acute endpoints during the last 40 years (Sorgeloos et al., 1978; Vanhaecke et al., 1980, 1981; Persoone and Castritsi-Catharios, 1989a,b) and thus few (and still not internationally standardized) chronic protocols exist.

Gebhardt (1976) evaluated the chronic effects of $\mathrm{Cd}, \mathrm{Cu}$, and $\mathrm{Hg}$ on A. salina growth and reproduction using a static renewal test with medium and food replacement every 3 days. Natural seawater from the Great Salt Lake (Utah, USA) was used as testing medium, and brine shrimp were fed with Dunaliella viridis. Adults were investigated for immobilization, newly hatched nauplii for growth and reproduction inhibition and eggs for hatching experiments. $\mathrm{Cu}$ and $\mathrm{Hg}$ did not affect growth and reproduction at concentrations below acute mortality. Mating occurred both in negative controls, and $\mathrm{Cu}$ and $\mathrm{Hg}$ spiked media when brine shrimps were approximately 15 days old. The maximum length reached was of about $8 \mathrm{~mm}$. Only Cd between 1.0 and $33 \mathrm{mg} / \mathrm{L}$ significantly inhibited growth and reproduction rate. Cd delayed mating of about two days compared to the controls. No mating occurred at $33 \mathrm{mg} / \mathrm{L}$ of $\mathrm{Cd}$, and no nauplii were produced during the experiment at this concentration.

Cunningham (1976) investigated the effects of Dimilin (TH 6040) on various brine shrimp life stages considering a static renewal test. Artemia cultures consumed algae present in the jars and were supplemented with a few drops of yeast suspension daily. Brine shrimp reproduction was evaluated exposing pairs of organisms and monitoring the subsequent number of produced nauplii. After 21 days, adult survivorship in the negative control was approximately $90 \%$ and declined to $70 \%$ after 28 days. After 40 days, survival dropped below $50 \%$ and all shrimps were dead after 80 days.

Browne and Wanigasekera (2000) measured reproductive performance and survival at nine temperature-salinity (T-S) combinations ( $T=15,24$ and $30^{\circ} \mathrm{C}$, and $S=60,120$ and $180 \%$ o) for four sexual (A. franciscana, A. salina, Artemia sinica and Artemia persimilis) and one parthenogenetic (A. parthenogenetica) species. During survival tests, Artemia was fed with yeast and Dunaliella tertiolecta once a day alternately. The number of dead individuals was counted after day $1,2,3,5,7,14$ and 21 . At sexual maturity, surviving individuals were transferred to jars containing brine and algal culture and monitored throughout their life cycle recording eleven life-history traits for each species (i.e. number of reproductive females, female pre-reproductive and reproductive periods, life-span for reproductive females, offspring per brood, broods per female, offspring per female, offspring per day per female, inter-brood interval, percentage of offspring encysted, and male life-span). All species showed the highest reproduction rate at $24{ }^{\circ} \mathrm{C}$ and at $120 \%$ o salinity for $A$. parthenogenetica, A. sinica and $A$. franciscana, and at $180 \%$ for $A$. salina and $A$. persimilis. Only at $24{ }^{\circ} \mathrm{C}$ and $120 \%$ salinity all species completed their whole life cycle. Results indicated that $A$. franciscana is both euryhaline and eurythermal reproducing at four T-S combinations. A. salina appeared cold-adapted and intolerant to low salinity (60\%) at any temperature. Reproductive performance and survival were optimal at $24{ }^{\circ} \mathrm{C}$ and $180 \%$ salinity.

Brix et al. (2003) determined the chronic (28 d) toxicity of As $\left(\mathrm{Na}_{3} \mathrm{AsO}_{4}\right)$ to $A$. franciscana measuring in triplicate the effects on growth, reproduction, and survival under intermittent flowthrough conditions considering a full life-cycle approach (Stephan et al., 1985). The study started considering $24 \mathrm{~h}$ old nauplii, and continued looking at the reproduction of the parental generation. Cysts were hatched in Great Salt Lake (Utah, USA) seawater $\left(27^{\circ} \mathrm{C}\right.$ and $28 \mathrm{~g} / \mathrm{L}$ of salts) that was used also as dilution water. After 11 d, brine shrimp sexually matured and began pairing for mating. At this time, a random subsample from each test concentration was collected and weighed (dry weight). Six adult pairs for each test concentration were then monitored for reproduction up to day 28 when surviving shrimps were measured for dry weight. Adult survival was the most sensitive endpoint, with growth and reproduction slightly less sensitive than survival. The no observed effect concentration (NOEC) for survival was $8 \mathrm{mg} / \mathrm{L}$, and the lowest observed effect concentration (LOEC) was $15 \mathrm{mg} / \mathrm{L}$ of dissolved As. Growth and reproduction LOEC values were $>56 \mathrm{mg} / \mathrm{L}$ that is the highest concentration tested. After the parental generation exposure to As, the F1 generation acclimated to As thus presenting a significant lower sensitivity than the parental generation.

Brix et al. (2004) investigated the use of Artemia for the derivation of a chronic site-specific water quality standard for Se in the Great Salt Lake (Utah, USA). The method previously described in Brix et al. (2003) was applied to A. franciscana exposed to different Se nominal concentrations $(0,3.8,7.5,15,30,60 \mathrm{mg} / \mathrm{L})$. Growth of the parental generation on day 11 and reproduction on day 21 were the two most sensitive endpoints for Artemia with respect to the other species evaluated (i.e. brine fly and hypersaline algae). Brine shrimp was the Great Salt Lake's most sensitive species resident, and the direct effects of Se on resident biota were not the critical exposure pathways in deriving a site-specific water quality discharge limit for the Great Salt Lake.

Savorelli et al. (2007) evaluated the conditions for 7-14 d bioassay with the brine shrimp Artemia, using $A$. franciscana and $A$. parthenogenetica. Exposures to SDS and oil dispersant were carried investigating somatic growth (i.e. carapace length from the top front of the head up to the caudal furca) and mortality. Algal food and toxicant were renewed three times a week. Test chambers were incubated at $25 \pm 1{ }^{\circ} \mathrm{C}, 900 \pm 100$ lux with a $14: 10 \mathrm{~h}$ light/dark photoperiod. Savorelli et al. (2007) proposed the use of II-III instar stage nauplii, Instant Ocean ${ }^{\circledR}$ salt mixture, and D. tertiolecta as food source for the $14 \mathrm{~d}$ toxicity test (sensitivity increases between $7 \mathrm{~d}$ and $14 \mathrm{~d}$ ) including a negative control success $\geq 80 \%$. Mortality was selected as endpoint for the proposed method being more sensitive than growth for both $A$. franciscana and A. parthenogenetica.

Manfra et al. (2012) proposed a video presenting on a step-bystep basis the $14 \mathrm{~d}$ toxicity test protocol of Savorelli et al. (2007) including testing materials (cysts, dilution media, algal culture and toxicant), cyst activation, preparation of testing toxicant (SDS) concentrations, preparation of testing media and food renewal. The video showed how to transfer nauplii $(n=10)$ from Petri dishes to test chambers in order to reduce dilution of testing solutions. After $14 \mathrm{~d}$, the number of surviving larvae was counted and $\mathrm{LC}_{50}$ calculated considering a threshold average mortality $\leq 20 \%$ and $\mathrm{LC}_{50}$ (SDS) $=8.0 \pm 5 \mathrm{mg} / \mathrm{L}$. Manfra et al. (2015a) considered a $14 \mathrm{~d}$ protocol with $A$. franciscana to investigate the toxicity of DEG as an anticorrosive agent frequently used in offshore oil drilling activities.

Manfra et al. (2016) published the results of the intercalibration exercise with SDS considering the $14 \mathrm{~d}$ toxicity test. As for the acute mortality test, eleven participating laboratories carried out three intercomparison assays calculating $\mathrm{LC}_{50}$ values and repeatability/reproducibility coefficients. Results complied with ISO (1996).

\section{Discussion}

After more than five decades of use in ecotoxicology, Artemia spp. demonstrated its ability mainly as pre-screening of toxic agents (Dvorak et al., 2012), thus Artemia spp. endpoints seem to respond to the market need of toxicity testing tools, even though no internationally standardised toxicity testing protocols currently exist according to OECD and ISO. 
Among the short-term toxicity tests, biomarkers and teratogenicity are the less popular endpoints with just few papers and citations probably due to their limited sensitivity. Behavioral endpoints, especially swimming inhibition, have been investigated only recently and seem to have a great potential in the near future, mainly because results can be acquired via image and video analysis taking into account both continuous and periodic observations of single or groups of animals. Hatching and acute mortality are the most common endpoints with a different level of on-going standardization process. Hatching ( $48 \mathrm{~h}$ static test) was intercalibrated at Italian level (Manfra et al., 2015b), while acute mortality (24 h static test) was intercalibrated on the basis of the available standard (APAT and IRSA-CNR, 2003) at Italian (Manfra et al., 2015b) as well as European level (Persoone et al., 1993). Both provided data on $\mathrm{CuSO}_{4}$ as reference toxicant. Among the long-term toxicity tests, the $14 \mathrm{~d}$ static-renewal mortality test was intercalibrated at Italian level (Manfra et al., 2015b) with SDS on the basis of Unichim protocol (2012).

To make Artemia spp. an official internationally recognized standard biological model in ecotoxicology and nanoecotoxicology, further efforts are necessary. The steps towards standardization should involve (i) national member (who then contacts ISO) upon a request by an industry sector or group for a standard; (ii) creation of a group of experts from all over the world negotiating all aspects of the standard, including its scope, key definitions and content; (iii) multi-stakeholder brainstorming and reviewing process including experts from the relevant industry, consumer associations, academia, non governmental organizations and government.

We highlight some tasks to be easily accomplished as a correct practice to ameliorate the feasibility, reliability and comparability of Artemia spp. toxicity data including all endpoints and potentially contributing to support official standardization. Authors did not report and reviewers did not require several key parameters. According to Tables S1, S2, 1-3, frequent omissions are present: $\mathrm{pH}$, aeration conditions (DO), amount of hatched cysts, feeding rate, test container, quality criteria (QC) for negative controls and reference toxicant. In Table S1, the acceptability threshold for cysts hatchability is repeatedly missing like as the reference toxicant. Hatching toxicity tests with Artemia spp. never provided saltwater $\mathrm{pH}$ values (Table S2), and, just in few cases, data about light conditions during exposure, and positive and negative controls quality criteria for acceptability. In Table 1 , the positive control is frequently absent like as the determination of EC50 values for the swimming velocity inhibition, thus limiting the comparability with other endpoints' sensitivity. Within the behavioral endpoints, a harmonization of protocols is strongly required also because authors proposed timeby-time specific approaches, apparatuses, tracking and recording devices. In Table 2, the database is quite complete probably because the $24 \mathrm{~h}$ short-term toxicity test is the most widespread. Missing information is generally related to the amount of hatched cysts and DO that represent interesting parameters to fully circumscribe the general test (pre-)conditions. Similarly, it was found for Table 3 where data about reference toxicants are very scarce too. Finally, it is not clear which is the cost effectiveness of each endpoint compared to its relative sensitivity and current readiness for the market.

In conclusion, we can provide the following order of easy-to-standardize toxicity tests: $24-48 \mathrm{~h}$ mortality $>14-28 \mathrm{~d}$ mortality $>$ hatching test $>$ behavioral endpoints $>$ biomarkers.

\section{Appendix A. Supplementary data}

Supplementary data associated with this article can be found, in the online version, at http://dx.doi.org/10.1016/j.ecolind.2016. 04.017.

\section{References}

APAT and IRSA-CNR, 2003. Metodi analitici per le acque (in Italian).

Alyürüc, H., Çavas, L., 2013. Toxicity of diuron and irgarol on the hatchability and early stage development of Artemia salina. Turk. J. Biol. 37, 151-157.

Alyuruk, H., Demir, G.K., Cavas, L., 2013. A video tracking based improvement of acute toxicity test on Artemia salina. Mar. Freshw. Behav. Physiol. 46, 251-266.

Artoxkit, M., 2014. Artemia Toxicity Screening Test for Estuarine and Marine Waters. Standard Operational Procedure. Microbiotests, Mariakerke-Gent.

Bagshaw, J.C., Rafiee, P., Matthews, C.O., MacRae, T.H., 1986. Cadmium and zinc reversibly arrest development of Artemia larvae. Bull. Environ. Contam. Toxicol. 37, 289-296.

Barahona, M.V., Sánchez-Fortún, S., 1999. Toxicity of carbamates to the brine shrimp Artemia salina and the effect of atropine: iso-ompa and 2-PAM on carbaryl toxicity. Environ. Pollut. 104, 469-476.

Barahona-Gomariz, M.V., Sanz-Barrera, F., Sánchez-Fortún, S., 1994. Acute toxicity of organic solvents on Artemia salina. Bull. Environ. Contam. Toxicol. 52 (5), 766-771.

Beattie, K.A., Ressler, J., Wiegand, C., Krause, E., Codd, G.A., Steinberg, C.E.W., Pflugmacher, S., 2003. Comparative effects and metabolism of two microcystins and nodularin in the brine shrimp Artemia salina. Aquat. Toxicol. $62,219-226$.

Bergami, E., Bocci, E., Vannuccini, M.L., Monopoli, M., Salvati, A., Dawson, K.A., Corsi, I., 2016. Nano-sized polystyrene affects feeding: behavior and physiology of brine shrimp Artemia franciscana larvae. Ecotoxicol. Environ. Saf. 123, 18-25.

Boone, E., Baas-Becking, L.G.M., 1931. Salt effects on eggs and nauplii of Artemia salina L. J. Gen. Physiol. 20, 753-763.

Brix, K.V., Cardwell, R.D., Adams, W.J., 2003. Chronic toxicity of arsenic to the Great Salt Lake brine shrimp, Artemia franciscana. Ecotoxicol. Environ. Saf. 54, 169-175.

Brix, K.V., Deforest, D.K., Cardwell, R.D., Adams, W.J., 2004. Derivation of a chronic site-specific water quality standard for selenium in the Great Salt Lake Utah, USA. Environ. Toxicol. Chem. 23, 606-612.

Brix, K.V., Gerdes, R.M., Adams, W.J., Grosell, M., 2006. Effect of copper, cadmium, and zinc on the hatching success of brine shrimp (Artemia franciscana). Arch. Environ. Contam. Toxicol. 51, 580-583.

Browne, T.A., 1980. Acute response versus reproductive performance in five strains of brine shrimp exposed to copper sulphate. Mar. Environ. Res. 3, 185-193.

Browne, R.A., Wanigasekera, G., 2000. Combined effects of salinity and temperature on survival and reproduction of five species of Artemia. J. Exp. Mar. Biol. Ecol. 244, 29-44.

Browne, R.A., Li, M., Wanigasekera, G., Simonek, S., Brownlee, D., Eiband, G., Cowan, J., 1991. Ecological, physiological and genetic divergence of sexual and asexual (diploid and polyploid) brine shrimp (Artemia). In: Menon, J. (Ed.), Advances in Ecology, vol. 1. Council of Research Integration, Trivandrum, India, pp. 41-52.

Bustos-Obregon, E., Vargas, Á., 2010. Chronic toxicity bioassay with populations of the crustacean Artemia salina exposed to the organophosphate diazinon. Biol. Res. 43 (3), 357-362.

Cáceres, A., López, B., González, S., Berger, I., Tada, I., Maki, J., 1998. Plants used in Guatemala for the treatment of protozoal Infections. I. Screening of activity to bacteria, fungi, and American trypanosomes of 13 native plants. J. Ethnopharmacol. 62, 195-202.

Caldwell, G.S., Bentley, M.G., Olive, P.J.W., 2003. The use of brine shrimp (Artemia salina) bioassay to assess the toxicity of diatom extract and short chain aldehydes. Toxicon 42, 301-306.

Callegaro, S., Minetto, D., Pojana, G., Bilanicová, D., Libralato, G., Volpi Ghirardini A., Hassellöv, M., Marcomini, A., 2015. Effects of alginate on stability and ecotoxicity of nano- $\mathrm{TiO}_{2}$ in artificial seawater. Ecotoxicol. Environm. Saf. 117, 107-114.

Carballo, J.L., Hernández-Inda, Z.L., Pérez, P., García-Grávalos, M.D., 2002. A comparison between two brine shrimpassays to detect in vitro cytotoxicity in marine natural products. BMC Biotechnol. 2, 17.

Castritsi-Catharios, J., Bourdaniotis, N., Persoone, G., 2007. A new simple method with high precision for determining the toxicity of antifouling paints on brine shrimp larvae (Artemia): first results. Chemosphere 67, 1127-1132.

Castritsi-Catharios, J., Zouganelis, G., Bourdaniotis, N., Koinis, S., 2012. ALDH activity of Artemia as a tool for the investigation of antifouling paints. In: Enzymes and Enzyme Activity: Structure and Mechanisms, Kinetics and Role in Disease (2012). Nova Science Publishers, Hauppauge, NY.

Castritsi-Catharios, J., Syriou, V., Miliou, H., Zouganelis, G.D., 2013. Toxicity effects of bisphenol A to the nauplii of the brine shrimp Artemia franciscana. J. Biol. Res. (Thessalon) 19, 38-45.

Castritsi-Catharios, J., Alambritis, G., Miliou, H., Cotou, E., Zouganelis, D., 2014. Comparative toxicity of tin free self-polishing copolymer antifouling paints and their inhibitory effects on larval development of a non-target organism. Mater. Sci. Appl. 5, 158-169.

Corsi, I., Cherr, G.N., Lenihan, H.S., Labille, J., Hassellov, M., Canesi, L., Dondero, F., Frenzilli, G., Hristozov, D., Puntes, V., Della Torre, C., Pinsino, A., Libralato, G., Marcomini, A., Sabbioni, E., Matranga, V., 2014. Common strategies and technologies for the ecosafety assessment and design of nanomaterials entering the marine environment. ACS Nano 8, 9694-9709.

Cunningham, P.A., 1976. Effects of dimilin (TH 6040) on reproduction in the brine shrimp, Artemia salina. Environ. Entomol. 5, 701-706.

Davenport, J., Healy, A., 2006. Relationship between medium salinity, body density, buoyancy and swimming in Artemia franciscana larvae: constraints on water column use? Hydrobiologia 556, 295-301. 
Day, R.M., Gupta, J.S., MacRae, T.H., 2003. A small heat shock/ $\alpha$-crystallin protein from encysted Artemia embryos suppresses tubulin denaturation. Cell Stress Chaperones 8, 183-193.

Delinger, L., 2002. Regulation of diapauses. Annu. Rev. Entomol. 47, 93-122.

Dvorak, P., Benova, K., Vitek, J., 2012. Alternative biotest on Artemia franciscana. Chapter 3 p. 51-74. In: Begum, Ghousia (Ed.), Ecotoxicology. InTech, Rijeka, Croatia, p. 146.

Espiritu, E.Q., Janssen, C.R., Persoone, G., 1995. Cyst-based toxicity tests VII: evaluation of the $1 \mathrm{~h}$ enzymatic inhibition test (Fluotox) with Artemia nauplii. Environ. Toxicol. Water Qual. 10, 25-34.

Faimali, M., Garaventa, F., Piazza, V., Corrà, C., Magillo, F., Pittore, M., Giacco, E. Gallus, L., Falugi, C., Tagliafierro, G., 2006. Swimming speed alteration of larvae of Balanus amphitrite as behavioural end-point for laboratory toxicological bioassays. Mar. Biol. 149, 87-96.

Favilla, M., Macchia, L., Gallo, A., Altomare, C., 2006. Toxicity assessment of metabolites of fungal biocontrol agents using two different (Artemia salina and Daphnia magna) invertebrate bioassays. Food Chem. Toxicol. 44 (11), 1922-1931.

Gambardella, C., Mesarič, T., Milivojević, T., Sepčić, K., Gallus, L., Carbone, S., Ferrando, S., Faimali, M., 2014. Effects of selected metal oxide nanoparticles on Artemia salina larvae: evaluation of mortality and behavioural and biochemica responses. Environ. Monit. Assess. 186, 4249-4259.

Garaventa, F., Gambardella, C., Di Fino, A., Pittore, M., Faimali, M., 2010. Swimming speed alteration of Artemia sp. and Brachionus plicatilis as a sub-lethal behavioural end-point for ecotoxicological surveys. Ecotoxicology 19, 512-519.

Gebhardt, K.A., 1976. Effects of Heavy Metals (Cadmium, Copper, and Mercury) on Reproduction, Growth and Survival of Brine Shrimp (Artemia salina). Utah State University, Logan, UT.

Go, E.C., Pandey, A.S., MacRae, T.H., 1990. Effect of inorganic mercury on the emergence and hatching of the brine shrimp Artemia franciscana. Mar. Biol. 107, 93-102.

Granade, H.R., Cheng, P.C., Doorenbos, N.J., 1976. Ciguatera, I: brine shrimp (Artemia salina L.) larval assay for ciguatera toxins. J. Pharm. Sci. 65, 1414-1415.

Grammou, A., Papadimitriou, C., Samaras, P., Vasara, E., Papadopoulos, A.I., 2011 Effect of municipal waste water effluent upon the expression of Glutathione S-transferase isoenzymes of brine shrimp Artemia. Chemosphere 84 (1), 105-109.

Grosch, D.S., 1980. Alterations to the reproductive performance of Artemia caused by antifouling paints, algaecides, and an aquatic herbicide. The Brine Shrimp Artemia, 201-211. In: Bioactive Natural Products Detection, Isolation, and Structural Determination from Steven M. Colegate, Russell J. Molyneux. CRC Press.

Guerra, R., 2001. Ecotoxicological and chemical evaluation of phenolic compounds in industrial effluents. Chemosphere 44, 1737-1747.

Guzzella, 1997. Saggio di tossicità acuta con Artemia sp. Biologia Ambientale 1. In Italian.

Hadjispyrou, S., Kungolos, A., Anagnostopoulos, A., 2001. Toxicity, bioaccumulation, and interactive effects of organotin: cadmium and chromium on Artemia franciscana. Ecotoxicol. Environ. Saf. 49, 179-186.

Huang, Y., Aldasoro, C.C.R., Persoone, G., Wlodkowic, D., 2015. Integrated microfluidic technology for sub-lethal and behavioral marine ecotoxicity biotests. Proc. Int. Soc. Opt. Eng. SPIE 9518, 10

ISO, 1996. Water Quality Determination of the Inhibition of the Mobility of Daphnia magna Straus (Cladocera, Crustacea). ISO 6341, International Standardization Organization, Geneva, Switzerland.

Jones, A.G., Ewing, C.M., Melvin, M.V., 1981. Biotechnology of solar saltfields. Hydrobiologia 82, 391-406.

Jorgensen, K.F., Jensen, K., 1977. Effect of copper(II) chloride on the hatching rate of Artemia salina (L.). Chemosphere 6, 287-291.

Katranitsas, A., Castritsi-Catharios, J., Persoone, G., 2003. The effects of a copper-based antifouling paint on mortality and enzymatic activity of a non-target marine organism. Mar. Pollut. Bull. 46, 1491-1494.

Kerster, H.W., Schaeffer, D.J., 1983. Brine shrimp (Artemia salina) nauplii as a teratogen test system. Ecotoxicol. Environ. Saf. 7, 342-349.

Kinne, O., 1971. Salinity: animals-invertebrates. Mar. Ecol. 1, 821-995.

Kissa, E., Moraitou-Apostolopoulou, M., Kiortsis, V., 1984. Effects of four heavy metals on survival and hatching rate of Artemia salina (L.). Arch. Hydrobiol. $102,255-264$

Kokkali, V., Katramados, I., Newman, J.D., 2011. Monitoring the effect of metal ions on the mobility of Artemia salina nauplii. Biosensors 1 (2), 36-45.

Koutsaftis, A., Aoyama, I., 2007. Toxicity of four antifouling biocides and their mixtures on the brine shrimp Artemia salina. Sci. Total Environ. 387 (1), $166-174$.

Koutsaftis, A., Aoyama, I., 2008. Toxicity of diuron and copper pyrithione on the brine shrimp Artemia franciscana: the effects of temperature and salinity. J Environ. Sci. Health Part A: Toxic/Hazard. Subst. Environ. Eng. 43, 1581-1585.

Krishnakurmar, P.K., Dineshbabu, A.P., Sasikumar, G., Bhat, G.S., 2007. Toxicity evaluation of treated refinery effluent using brine shrimp (Artemia salina) eggs and larval bioassay. Fish. Technol. 44, 85-92.

Kuwabara, K., Nakamura, A., Kashimoto, T., 1980. Effect of petroleum oil, pesticides, PCBs and other environmental contaminants on the hatchability of Artemia salina dry eggs. Bull. Environ. Contam. Toxicol. 25, 69-74.

Larsen, P.S., Madsen, C.V., Riisgård, H.U., 2008. Effect of temperature and viscosity on swimming velocity of the copepod Acartia tonsa: brine shrimp Artemia salina and rotifer Brachionus plicatilis. Aquat. Biol. 4, 47-54.
Léger, P., Bengston, D.A., Simpson, K.L., Sorgeloos, P., 1986. The use and nutritional value of Artemia as a food source. In: Barnes, H., Barnes, M. (Eds.) Oceanography and Marine Biology. An Annual Review. Aberdeen University Press, Aberdeen.

Leis, M., Manfra, L., Taddia, L., Chicca, M., Trentini, P., Savorelli, F., 2014. A comparative toxicity study between an autochthonous Artemia and a non native invasive species. Ecotoxicology 23 (6), 1143-1145.

Libralato, G., 2014. The case of Artemia spp. in nanoecotoxicology. Mar. Environ. Res. 101, 38-43

Libralato, G., Losso, C., Volpi Ghirardini, A., 2007. Toxicity of untreated wood leachates towards two saltwater organisms (Crassostrea gigas and Artemia franciscana). J. Hazard. Mater. 144, 590-593.

Libralato, G., Volpi Ghirardini, A., Avezzù, F., 2010a. Seawater ecotoxicity of monoethanolamine, diethanolamine and triethanolamine. J. Hazard. Mater. $176,535-539$

Libralato, G., Volpi Ghirardini, A., Avezzù, F., 2010b. How toxic is toxic: a proposa for wastewater toxicity hazard assessment. Ecotoxicol. Environ. Saf. 73, $1602-1611$

Little, E.E., Finger, S.E., 1990. Swimming behaviour as an indicator of sublethal toxicity in fish. Environ. Toxicol. Chem. 9, 13-19.

MacRae, T.H., Pandey, A.S., 1991. Effect of metals on early life stages of the brine shrimp, Artemia: a development toxicity assay. Arch. Environ. Contam. Toxicol. 20, 247-252.

Manfra, L., De Nicola, E., Maggi, C., Zambianchi, E., Caramiello, D., Toscano, A., Cianelli, D., Cicero, A.M., 2011. Exposure of rotifers, crustaceans and sea urchins to produced formation waters and seawaters in the Mediterranean Sea. J. Mar. Biol. Assoc. U.K. 91 (01), 155-161.

Manfra, L., Maggi, C., Bianchi, J., Mannozzi, M., Faraponova, O., Mariani, L., Onorati, F., Tornambè, A., Lamberti, C.V., Magaletti, E., 2010. Toxicity evaluation of produced formation waters after filtration treatment. Natural Sci. 2 (01), 33

Manfra, L., Savorelli, F., Pisapia, M., Magaletti, E., Cicero, A.M., 2012. Long-term lethal toxicity test with the crustacean Artemia franciscana. JoVE 62, 2182-2185

Manfra, L., Tornambè, A., Savorelli, F., Rotini, A., Canepa, S., Mannozzi, M., Cicero, A.M., 2015a. Ecotoxicity of diethylene glycol and risk assessment for marine environment. J. Hazard. Mater. 284, 130-135.

Manfra, L., Savorelli, F., Di Lorenzo, B., Libralato, G., Comin, S., Conti, D., Floris, B., Francese, M., Gallo, M.L., Gartner, I., Guida, M., Leoni, T., Marino, G., Martelli, F., Palazzi, D., Prato, E., Righini, P., Rossi, E., Volpi Ghirardini, A., Migliore, L., 2015b. Intercalibration of ecotoxicity testing protocols with Artemia franciscana. Ecol. Indic. 57, 41-47.

Manfra, L., Canepa, S., Piazza, V., Faimali, M., 2016. Lethal and sublethal endpoints observed for Artemia exposed to two reference toxicants and an ecotoxicological concern organic compound. Ecotoxicol. Environ. Saf. 123, 60-64.

Medlyn, R.A., 1980. Susceptibility of four geographical strains of adult Artemia to Ptychodiscus brevis toxin(s). The Brine Shrimp Artemia. In: Persoone, G., Sorgeloos, P., Roels, O., Jaspers, E. (Eds.), The Brine Shrimps Artemia Ecology, Culturing, Use in Aquaculture, vol. 3. Universa Press, Wetteren. Belgium, 1, 225-231.

Miah, F., Ahmed, K., Quddus, M.M.A., 2010. Characterization and functional role of heat shock protein 26 in Artemia. World J. Zool. 5, 282-294.

Michael, A.S., Thompson, C.B., Abramovitz, M., 1956. Artemia salina as a test organism for bioassay. Science 123, 464 .

Migliore, L., Dojmi di Delupis, G., Cappellaro, H., Brambilla, G., 1993a. Drugs in aquaculture: monitoring of contamination and proposal for its reduction by the use of Artemia salina (L.) in lakes and reservoirs. In: Giussani, G., Callieri, C. (Eds.), Strategies for Lake Ecosystems Beyond 2000. Griggi G.M., Baveno (No), Italy, pp. 291-294

Migliore, L., Brambilla, G., Grassitelli, A., Di Delupis, G., 1993b. Toxicity and bioaccumulation of sulphadimethoxine in Artemia (Crustacea, Anostraca). Int. J. Salt Lake Res. 2, 141-152.

Migliore, L., Civitareale, C., Brambilla, G., Dojmi di Delupis, G., 1997. Toxicity of several important antibiotics to Artemia. Water Res. 31, 1801-1806.

Migliore, L., Rotini, A., Randazzo, D., Albanese, N.N., Giallongo, A., 2007. Phenols content and 2-D electrophoresis protein pattern to monitor Posidonia meadows health state. BMC Ecol. 7, 6.

Minetto, D., Libralato, G., Volpi Ghirardini, A., 2014. Ecotoxicity of engineered $\mathrm{TiO}_{2}$ nanoparticles to saltwater organisms: An overview. Environ. Int. 66, 18-27.

Morris, J.E., Afzelius, B.A., 1967. The structure of the shell and outer membranes in encysted Artemia salina embryos during cryptobiosis and development. J. Ultrastruct. Res. 20 (3-4), 244-259.

Nakanishi, Y.H., Iwasaki, T., Okigaki, T., Kato, H., 1962. Cytological studies of Artemia salina I: embryonic development without cell multiplication after the blastula stage in encysted dry eggs. Annot. Zool. Jap. 35, 223-228.

Neumeyer, C.H., Gerlach, J.L., Ruggiero, K.M., Covi, J.A., 2014. A novel model of early development in the brine shrimp, Artemia franciscana, and its use in assessing the effects of environmental variables on development, emergence, and hatching. J. Morphol. 276, 342-360.

Nováková, J., Daňová, D., Strišková, K., Hromada, R., Mičková, H., Rabišková, M. 2007. Zinc and cadmium toxicity using a biotest with Artemia franciscana. Acta Vet. Brno 76 (4), 635-642

Nunes, B.S., Carvalho, F.D., Guilhermino, L.M., Van Stappen, G., 2006a. Use of the genus Artemia in ecotoxicity testing. Environ. Pollut. 144, 453-462. 
Nunes, B., Carvalho, F., Guilhermino, L., 2006b. Effects of widely used pharmaceuticals and a detergent on oxidative stress biomarkers of the crustacean Artemia parthenogenetica. Chemosphere 62, 581-594.

O'Connell, P.A., Pinto, D.M., Chisholm, K.A., MacRae, T.H., 2006. Characterization of the microtubule proteome during post-diapause development of Artemia franciscana. Biochim. Biophys. Acta 1764, 920-928.

Okamura, H., Aoyama, I., Liu, D., Maguire, R.J., Pacepavicius, G.J., Lau, Y.L., 2000. Fate and ecotoxicity of the new antifouling compound Irgarol 1051 in the aquatic environment. Water Res. 34, 3523-3530.

Olson, C.S., 1979. Timing of developmental events in Artemia salina (L.) (Anostraca). Cruscaceana 36, 302-308.

Pane, L., Agrone, C., Giacco, E., Somà, A., Mariottini, G.L., 2012. Utilization of marine crustaceans as study models: a new approach in marine ecotoxicology for European (REACH) regulation 91. Chapter 5, p. 91-107. In: Begum, G. (Ed.), Ecotoxicology. InTech, Rijeka, Croatia, p. 146.

Peakall, D., 1992. Animal biomarkers as pollution indicators. In: Depledge, M.H., Sanders, B. (Eds.), Biomarkers of the Nervous System. Chapman \& Hall, London.

Persoone, G., Castritsi-Catharios, J., 1989a. A simple bioassay with Artemia larvae to determine the acute toxicity of antifouling paints. Water Res. 23, 893-897.

Persoone, G., Sorgeloos, P., 1980. General aspects of the ecology and biogeography of Artemia. In: Persoone, G., Sorgeloos. Roels, P.O., Jaspers, E. (Eds.), The Brine Shrimps Artemia Ecology, Culturing, Use in Aquaculture, vol. 3. Universa Press, Wetteren. Belgium, pp. 3-24.

Persoone, G., Blaise, Chr., Snell, T., Janssen, C., van Steertegem, M., 1993. Cyst-based toxicity test: II-report on an international intercalibration exercise with three cost-effective toxkits. Angew. Zool. 1, 17-34.

Persoone, G., Castritsi-Catharios, J., 1989b. A simple bioassay with Artemia larvae to determine the acute toxicity of antifouling paints. Water Res. 23, 893-897.

Peteros, N.P., Uy, M.M., 2010. Antioxidant and cytotoxic activities and phytochemical screening of four Philippine medicinal plants. J. Med. Plants Res. 4, 407-414.

Petrucci, F., Caimi, S., Mura, G., Caroli, S., 1995. Artemia as a test organism of environmental contamination by trace elements. Microchem. J. 51, 181-186.

Pimentel, M.F., De Lima, D.P., Martins, L.R., Beatriz, A., Santaella, S.T., Lotufo, L.V.C. 2009. Ecotoxicological analysis of cashew nut industry effluents, specifically two of its major phenolic components, cardol and cardanol. Pan-Am. J. Aquat. Sci. 4 (3), 363-368.

Prato, E., Biandolino, F., Bisci, A.P., Caroppo, C., 2011. Preliminary assessment of Ostreopsis cfr. ovata acute toxicity by using a battery bioassay. Chem. Ecol., $117-125$.

Rafiee, P., Matthews, C.O., Bagshaw, J.C., MacRae, T.H., 1986. Reversible arrest of Artemia development by cadmium. Can. J. Zool. 64, 1633-1641.

Rotini, A., Anello, L., Di Bernardo, M., Giallongo, A., Valiante, L., Migliore, L., 2013. Comparative analysis of bed density: total phenol content and protein expression pattern in Posidonia oceanica (L.) delile. Open Ecol. J. 3, 438-444

Rotini, A., Manfra, L., Canepa, S., Tornambè, A., Migliore, L., 2015. Can Artemia hatching assay be a (sensitive) alternative tool to acute toxicity test? Bull. Environ. Contam. Toxicol. 95, 745-751.

Sarabia, R., Varó, I., Torreblanca, A., Del Ramo, J.J., Pastor, A., Amat, F., Díaz-Mayans, J., 1998a. Accumulation of cadmium in several strains of Artemia. Cuadernos de Investigación Biológica 20, 435-438.

Sarabia, R., Torreblanca, A., Del Ramo, J.J., Díaz-Mayans, J., 1998b. Effects of low mercury concentration exposure on hatching, growth and survival in the Artemia strain La Mata parthenogenetic diploid. Comp. Biochem. Physiol. A Mol. Integr. Physiol. 120, 93-97.

Sarabia, R., Del Ramo, J., Varó, I., Díaz-Mayans, J., Torreblanca, A., 2002. Comparing the acute exposure to cadmium toxicity of nauplii from different populations of Artemia. Environ. Toxicol. Chem. 21, 437-444.

Sarabia, R., Del Ramo, J., Diaz-Mavans, J., Torreblanca, A., 2003. Development and reproductive effects of low cadmium concentration on Artemia parthenogenetica. J. Environ. Sci. Health A Toxicol. Hazard. Subst. Environ. Eng. 38, 1065-1071.

Sarabia, R., Varó, I., Amat, F., Pastor, A., Del Ramo, J.J., Díaz-Mayans, J., Torreblanca, A., 2006. Comparative toxicokinetics of cadmium in Artemia. Arch. Environ. Contam. Toxicol. 50, 111-120.

Sarabia, R., Del Ramo, J., Varó, I., Díaz-Mayans, J., Torreblanca, A., 2008. Sublethal zinc exposure has a detrimental effect on reproductive performance but not on the cyst hatching success of Artemia partenogenetica. Sci. Total Environ. 398, 48-52.

Savorelli, F., Palazzi, D., Gorbi, G., Invidia, M., Sei, S., Magaletti, E., Manfra, L., Gelli, F., 2007. Messa a punto di una metodologia di saggio a 14 giorni su Artemia franciscana e A partenogenetica. Biologia Ambientale 21, 1-10 (in Italian).

Sirdaarta, J., Cock, I.E., 2008. Vitamin E and Trolox ${ }^{\mathrm{TM}}$ reduce toxicity of Aloe barbadensis Miller juice in Artemia franciscana nauplii but individually are toxic at high concentrations. Internet J. Toxicol. 5, 1.

Sirdaarta, J., Cock, I.E., 2010. Effect of Aloe barbadensis Miller juice on oxidative stress biomarkers in aerobic cells using Artemia franciscana as a model. Phytother. Res. 24 (3), 360-364.

Solis, P.N., Wright, C.W., Anderson, M.M., Gupta, M.P., Phillipson, J.D., 1993. A microwell cytotoxicity assay using Artemia salina (brine shrimp). Planta Med. 59, 250-252.

Sorgeloos, P., Bossuyt, E., Laviña, E., Baeza-Mesa, M., Persoone, G., 1977. Decapsulation of Artemia cysts: a simple technique for the improvement of the use of brine shrimp in aquaculture. Aquaculture 12, 311-315.
Sorgeloos, P., Remiche-Van Der Wielen, C., Persoone, G., 1978. The use of Artemia nauplii for toxicity tests-a critical analysis. Ecotoxicol. Environ. Saf. 2, 249-255.

Stephan, C.E., Mount, D.I., Hansen, D.J., Gentile, J.H., Chapman, G.A., Brungs, W.A., 1985. Guidelines for Deriving Numerical National Water Quality Criteria for the Protection of Aquatic Organisms and their Uses. US Environmental Protection Agency, Environmental Research Laboratory, Duluth, MN.

Stewart, S., Schurr, K., 1980. Effects of asbestos on Artemia survival. In: Persoone, G., Sorgeloos, P., Roels, O., Jaspers, E. (Eds.), The Brine Shrimps Artemia Ecology, Culturing, Use in Aquaculture, vol. 3. Universa Press, Wetteren. Belgium, 1, 234-251.

Thomas, H.M., 2003. Molecular chaperones, stress resistance and development in Artemia franciscana. Semin. Cell Dev. Biol. 14, 251-258.

Toğulga, M., 1998. The short-term toxicity of two toxicants to Artemia nauplii. Turk. J. Zool. 22 (3), 259-266.

Tong, Z., Hongjun, J., Huailan, Z., 1996. Quality criteria of acrylonitrile for the protection of aquatic life in China. Chemosphere 32, 2083-2093.

Tornambè, A., Manfra, L., Mariani, L., Faraponova, O., Onorati, F., Savorelli, F., Cicero A.M., Virno Lamberti, C., Magaletti, E., 2012. Toxicity evaluation of diethylene glycol and its combined effects with produced waters of off-shore gas platforms in the Adriatic Sea (Italy): bioassays with marine/estuarine species. Mar. Environ. Res. 77, 141-149.

Treece, G.D., 2000. Artemia production for marine larval fish culture. Southern Regional Aquaculture Center Publication No. 702.

Triantaphyllidis, G.V., Poulopoulou, K., Abatzopoulos, T.J., Pérez, C.A.P., Sorgeloos, P., 1995. International study on Artemia XLIX. Salinity effects on survival, maturity, growth, biometrics, reproductive and lifespan characteristics of a bisexual and a parthenogenetic population of Artemia. Hydrobiologia 302 (3), 215-227.

Triantaphyllidis, G.V., Abatzopoulos, T.J., Sorgeloos, P., 1998. Review of the biogeography of the genus Artemia (Crustacea, Anostraca). J. Biogeogr. 25, 213-226.

Trieff, N.M., 1980. Toxicity of heavy metals, oils and other organics on Artemia. In: Persoone, G., Sorgeloos, P., Roels, O., Jaspers, E. (Eds.), The Brine Shrimps Artemia Ecology, Culturing, Use in Aquaculture, vol. 3. Universa Press, Wetteren. Belgium, pp. 253-262.

Unichim, 2012. Qualità dell'acqua - Determinazione della tossicità letale a 14 giorni con Artemia franciscana (Crustacea: Anostraca). M.U. 2244:12. In Italian.

Vanhaecke, P., Persoone, G., 1981. Report on an intercalibration exercise on a short-term standard toxicity test with Artemia nauplii, 370-376.

Vanhaecke, P., Persoone, G., 1984. The ARC-test: a standardized short-term routine toxicity test with Artemia nauplii. In: Persoone, G., Jaspers, E., Claus, C. (Eds.), Ecotoxicological Testing for Marine Environment, vol. 2. State Univ. Ghent and Inst. Mar. Scient. Res, Bredene, pp. 143-157.

Vanhaecke, P., Sorgeloos, P., 1989. International study on Artemia. XLVII. The effect of temperature on cyst hatching: larval survival and biomass production for different geographical strains of brine shrimp Artemia spp. Ann. Soc. R. Zool. Belgium 119, 7-23.

Vanhaecke, P., Persoone, G., Claus, C., Sorgeloos, P., 1980. Research on the development of a short-term standard toxicity test with the Artemia nauplii. In: Persoone, G., Sorgeloos, P., Roels, O., Jaspers, E. (Eds.), The Brine Shrimp Artemia: Morphology, Genetics, Radiobiology, Toxicology, vol. 1. Universa Press, Wetteren, pp. 263-285.

Vanhaecke, P., Persoone, G., Claus, C., Sorgeloos, P., 1981. Proposal for a short-term toxicity test with Artemia nauplii. Ecotoxicol. Environ. Saf. 5 (3), 382-387.

Van Steertegem, M., Persoone, G., 1993a. Cyst-based toxicity tests: V. development and critical evaluation of standardized toxicity tests with the brine shrimp Artemia (Anostraca, Crustacea). In: Soares, A., Calow, P. (Eds.), Progress in Standardization of Aquatic Toxicity Tests. CRC Press.

Van Steertegem, M., Persoone, G., 1993b. Cyst-based toxicity tests V. development and critical evaluation of standardized toxicity tests with the brine shrimp Artemia (anostraca, Crustacea). In: Soares, A.M.V.M., Calow, P. (Eds.), Progress in Standardization of Aquatic Toxicity Tests. Lewis Publishers, pp. 81-97.

Varó, I., Taylor, A.C., Ferrando, M.D., Amat, F., 1997. Effect of endosulfan pesticide on the oxygen consumption rates of nauplii of different Spanish strains of Artemia. J. Environ. Sci. Health B: Pestic. Food Contam. Agric. Wastes 32, 363-375.

Varó, I., Navarro, J.C., Amat, F., Guilhermino, L., 2002. Characterisation of cholinesterases and evaluation of the inhibitory potential of chlorpyrifos and dichlorvos to Artemia salina and Artemia parthenogenetica. Chemosphere 48, 563-569.

Venkateswara Rao, J., Kavitha, P., Jakka, N.M., Sridhar, V., Usman, P.K., 2007. Toxicity of organophosphates on morphology and locomotor behavior in brine shrimp Artemia salina. Arch. Environ. Contam.Toxicol. 53, 225-232.

Veni, T., Pushpanathan, T., 2014. Comparison of the Artemia salina and Artemia fransiscana bioassays for toxicity of Indian medicinal plants. J. Coast. Life Med. 2 (6), 453-457, http://dx.doi.org/10.12980/JCLM.2.201414J29.

Vezie, C., Sivonen, K., Brient, L., Bertru, G., Lefeuvre, J.C., 1996. Development of toxic cyanobacteria in western france-detection of toxicity with Artemia salina tests. Ann. Limnol. Int. J. Limnol. 32, 123-128.

Vismara, C., 1998. Effects of methanol, ethanol and n-propanol on development of Artemia salina cysts. Chemosphere 37, 3027-3034.

Widdows, J., 1998. Marine and estuarine invertebrate toxicity tests. In: Calow, Peter (Ed.), Handbook of Ecotoxicology. Blackwell Science, Oxford (UK), pp. 51-65, ISBN 978-0-632- 04933-2. 
Williams, T.A., 1994a. Locomotion in developing Artemia larvae: mechanical analysis of antennal propulsors based on large-scale physical models. Biol. Bull. 187, 156-163.

Williams, T.A., 1994b. A Model of rowing propulsion and the ontogeny of locomotion in Artemia larvae. Biol. Bull. 187, 164-173.

Xu, X., Lu, Y., Zhang, D., Wang, Y., Zhou, X., Xu, H., Mei, Y., 2015. Toxic assessment of triclosan and triclocarban on Artemia salina. Bull. Environ. Contam. Toxicol. $95,728-733$.
Zillioux, E.J., Foulk, H.R., Prager, J.C., Cardin, J.A., 1973. Using Artemia to assay oil dispersant toxicities. J. Water Pollut. Control Fed. 45, 2389-2396.

Zulkifli, S.Z., Aziz, F.Z.A., Ajis, S.Z.M., Ismail, A., 2014. Nauplii of brine shrimp

(Artemia salina) as a potential toxicity testing organism for heavy metals

contamination. In: From Sources to Solution. Springer, Singapore, pp. 233-237. 